\title{
Boundary Harnack principle for Brownian motions with measure-valued drifts in bounded Lipschitz domains
}

\author{
Panki Kim • Renming Song
}

Received: 1 September 2005 / Published online: 5 May 2007

(C) Springer-Verlag 2007

\begin{abstract}
Let $\mu=\left(\mu^{1}, \ldots, \mu^{d}\right)$ be such that each $\mu^{i}$ is a signed measure on $\mathbf{R}^{d}$ belonging to the Kato class $\mathbf{K}_{d, 1}$. A Brownian motion in $\mathbf{R}^{d}$ with drift $\mu$ is a diffusion process in $\mathbf{R}^{d}$ whose generator can be informally written as $\frac{1}{2} \Delta+\mu \cdot \nabla$. When each $\mu^{i}$ is given by $U^{i}(x) d x$ for some function $U^{i}$, a Brownian motion with drift $\mu$ is a diffusion in $\mathbf{R}^{d}$ with generator $\frac{1}{2} \Delta+U \cdot \nabla$. In Kim and Song (Ill J Math 50(3):635-688, 2006), some properties of Brownian motions with measure-value drifts in bounded smooth domains were discussed. In this paper we prove a scale invariant boundary Harnack principle for the positive harmonic functions of Brownian motions with measure-value drifts in bounded Lipschitz domains. We also show that the Martin boundary and the minimal Martin boundary with respect to Brownian motions with measure-valued drifts coincide with the Euclidean boundary for bounded Lipschitz domains. The results of this paper are also true for diffusions with measure-valued drifts, that is, when $\Delta$ is replaced by a uniformly elliptic divergence form operator $\sum_{i, j=1}^{d} \partial_{i}\left(a_{i j} \partial_{j}\right)$ with $C^{1}$ coefficients or a uniformly elliptic non-divergence form operator $\sum_{i, j=1}^{d} a_{i j} \partial_{i} \partial_{j}$ with $C^{1}$ coefficients.
\end{abstract}

Mathematics Subject Classification (2000) Primary 31B25 · 60J45; Secondary $60 \mathrm{~J} 60 \cdot 31 \mathrm{C} 35$

The research of R. Song is supported in part by a joint US-Croatia grant INT 0302167. The research of P. Kim is supported by Research Settlement Fund for the new faculty of Seoul National University.

P. Kim $(\bowtie)$

Department of Mathematics, Seoul National University, Seoul 151-742, Republic of Korea e-mail: pkim@snu.ac.kr

R. Song

Department of Mathematics, University of Illinois, Urbana, IL 61801, USA

e-mail: rsong@math.uiuc.edu 


\section{Introduction}

The boundary Harnack principle in Lipschitz domains was first proved independently for the Laplacian $\Delta$ in $[1,14,26]$. Later the boundary Harnack principle was extended to uniformly elliptic operators with bounded coefficients in $[8,16]$ (see [4] for an extension to more general domains using probabilistic methods).

The main purpose of this paper is to prove a boundary Harnack principle in bounded Lipschitz domains for diffusions with measure-valued drifts. We will concentrate on Brownian motions with measure-valued drifts. The assumption on the drift is that each component of the drift belongs to the Kato class $\mathbf{K}_{d, 1}$ (see below for the definition). Our main results generalize the corresponding results of $[8,16]$ where only bounded drifts were considered.

In this paper, we always assume that $d \geq 3$. Suppose $\mu=\left(\mu^{1}, \ldots, \mu^{d}\right)$ is such that each $\mu^{i}$ is a signed measure on $\mathbf{R}^{d}$ belonging to the Kato class $\mathbf{K}_{d, 1}$, that is

$$
\lim _{r \downarrow 0} \sup _{x \in \mathbf{R}^{d}} \int_{|x-y| \leq r} \frac{\left|\mu^{i}\right|(d y)}{|x-y|^{d-1}}=0, \quad 1 \leq i \leq d .
$$

Informally, a Brownian motion in $\mathbf{R}^{d}$ with drift $\mu$ is a diffusion process in $\mathbf{R}^{d}$ with generator $\frac{1}{2} \Delta+\mu \cdot \nabla$. When each $\mu^{i}$ is given by $U^{i}(x) d x$ for some function $U^{i}$, a Brownian motion with drift $\mu$ is a diffusion in $\mathbf{R}^{d}$ with generator $\frac{1}{2} \Delta+U \cdot \nabla$ and it is a solution to the SDE

$$
d X_{t}=d W_{t}+U\left(X_{t}\right) \cdot d t
$$

To recall the precise definition of a Brownian motion with drift $\mu$ in $\mathbf{K}_{d, 1}$, we fix a non-negative smooth radial function $\varphi(x)$ in $\mathbf{R}^{d}$ with $\operatorname{supp}[\varphi] \subset B(0,1)$ and $\int \varphi(x) d x=1$. For any positive integer $n$, we put $\varphi_{n}(x)=2^{\text {nd }} \varphi\left(2^{n} x\right)$. For $1 \leq i \leq d$, define

$$
U_{n}^{i}(x)=\int \varphi_{n}(x-y) \mu^{i}(d y) .
$$

Put $U_{n}(x)=\left(U_{n}^{1}(x), \ldots, U_{n}^{d}(x)\right)$. The following definition is taken from [6].

Definition 1.1 Suppose $\mu=\left(\mu^{1}, \ldots, \mu^{d}\right)$ is such that each $\mu^{i}$ is a signed measure on $\mathbf{R}^{d}$ belonging to the Kato class $\mathbf{K}_{d, 1}$. A Brownian motion with drift $\mu$ is a family of probability measures $\left\{\mathbf{P}_{x}: x \in \mathbf{R}^{d}\right\}$ on $C\left([0, \infty), \mathbf{R}^{d}\right)$, the space of continuous $\mathbf{R}^{d}$-valued functions on $[0, \infty)$, such that under each $\mathbf{P}_{x}$ we have

$$
X_{t}=x+W_{t}+A_{t}
$$

where

(a) $\quad A_{t}=\lim _{n \rightarrow \infty} \int_{0}^{t} U_{n}\left(X_{s}\right) d s$ uniformly in $t$ over finite intervals, where the convergence is in probability; 
(b) there exists a subsequence $\left\{n_{k}\right\}$ such that

$$
\sup _{k} \int_{0}^{t}\left|U_{n_{k}}\left(X_{S}\right)\right| d s<\infty
$$

almost surely for each $t>0$;

(c) $W_{t}$ is a standard Brownian motion in $\mathbf{R}^{d}$ starting from the origin.

In this paper, we will fix a $\mu=\left(\mu^{1}, \ldots, \mu^{d}\right)$ with each $\mu^{i} \in \mathbf{K}_{d, 1}$ and use $X$ to denote a Brownian motion with drift $\mu$ defined above. The existence and uniqueness of $X$ were established in [6]. In fact, $X$ was shown to be a Feller process.

Bass and Chen raised the following question in [6]: do the Harnack principle and the boundary Harnack principle hold for the positive harmonic functions of $X$ ? Recall that a non-negative function $f$ on a domain $D$ is said to be harmonic in $D$ with respect to $X$ if for every relatively compact open subset $B$ of $D$ with $\bar{B} \subset D$,

$$
f(x)=\mathbf{E}_{x}\left[f\left(X_{\tau_{B}}\right)\right], \quad x \in D
$$

where $\tau_{B}=\inf \left\{s>0: X_{s} \notin B\right\}$.

In [21], we showed that $X$ has a density $q(t, x, y)$ which is continuous on $(0, \infty) \times$ $\mathbf{R}^{d} \times \mathbf{R}^{d}$ and that there exist positive constants $C_{i}, i=1, \ldots, 9$ such that

$$
C_{1} e^{-C_{2} t} t^{-\frac{d}{2}} e^{-\frac{C_{3}|x-y|^{2}}{2 t}} \leq q(t, x, y) \leq C_{4} e^{C_{5} t} t^{-\frac{d}{2}} e^{-\frac{C_{6}|x-y|^{2}}{2 t}}
$$

and

$$
\left|\nabla_{x} q(t, x, y)\right| \leq C_{7} e^{C_{8} t} t^{-\frac{d+1}{2}} e^{-\frac{C_{9}|x-y|^{2}}{2 t}}
$$

for all $(t, x, y) \in(0, \infty) \times \mathbf{R}^{d} \times \mathbf{R}^{d}$. We also showed that, for every bounded $C^{1,1}$ domain $D, X^{D}$ (the process obtained by killing $X$ upon exiting from $D$ ) has a density $q^{D}$ which is continuous on $(0, \infty) \times D \times D$ and that for any $T>0$, there exist positive constants $C_{i}, i=10, \ldots, 14$, such that

$$
C_{10} \psi_{D}(t, x, y) t^{-\frac{d}{2}} e^{-\frac{C_{11}|x-y|^{2}}{t}} \leq q^{D}(t, x, y) \leq C_{12} \psi_{D}(t, x, y) t^{-\frac{d}{2}} e^{-\frac{C_{13}|x-y|^{2}}{t}}
$$

and

$$
\left|\nabla_{x} q^{D}(t, x, y)\right| \leq C_{14}\left(1 \wedge \frac{\rho(y)}{\sqrt{t}}\right) t^{-\frac{d+1}{2}} e^{-\frac{C_{13}|x-y|^{2}}{t}}
$$

for all $(t, x, y) \in(0, T] \times D \times D$, where $a \wedge b:=\min \{a, b\}, \rho(x)$ is the distance between $x$ and $\partial D$ and

$$
\psi_{D}(t, x, y):=\left(1 \wedge \frac{\rho(x)}{\sqrt{t}}\right)\left(1 \wedge \frac{\rho(y)}{\sqrt{t}}\right) .
$$

(See Sect. 4 of [18] for estimates of heat kernels of the Schrödinger-type equations for $X^{D}$.) By using these estimates, we established in [21] that the Harnack principle holds 
for the positive harmonic functions of $X$ and that the boundary Harnack principle holds for the positive harmonic functions of $X$ in bounded $C^{1,1}$ domains.

However, the boundary Harnack principle established in [21] is not scale invariant and the domains are assumed to be bounded $C^{1,1}$. The purpose of this paper is to prove a scale invariant version of the boundary Harnack principle for bounded Lipschitz domains and to identify the Martin boundary.

A nice framework for the potential theory of strong Markov processes without the duality assumption was proposed in [13]. In this paper, we first show that Brownian motions with measure-valued drifts fit nicely into this framework. Then we revisit the Green function estimates obtained in [21] and show that, in fact, the Green function for a Brownian motion with measure-valued drift is uniformly comparable to that of a Brownian motion in small sets. Using an argument similar to that of the proof of Theorem 2.2 in [9], we prove that the comparability of Green functions implies the comparability of harmonic measures.

Bass and Burdzy [3,5] introduced a probabilistic method, the so-called box method, to prove the boundary Harnack principle. In this paper, by using this method and combining the uniform comparability of harmonic measures with the results of [21], we prove a Carleson type estimate and a scale invariant boundary Harnack principle for Brownian motions with measure-valued drifts in bounded Lipschitz domains.

Finally, we apply the Carleson type estimate and the boundary Harnack principle together with the results of [21] to identify the Martin boundary with respect to Brownian motions with measure-valued drifts in bounded Lipschitz domains. Unlike the case of symmetric processes, identifying the Martin boundary with the Euclidean boundary is very delicate in the present case. We show that if $D$ is a bounded Lipschitz domain, there exists a homeomorphism between the Martin compactification and $\bar{D}$ which is an identity map in $D$. Unlike [21], there are no sharp estimates on the Green functions of Brownian motions with measure-valued drifts in bounded Lipschitz domains. Instead we use the Carleson type estimate and the boundary Harnack principle. The scale invariant property of our boundary Harnack principle is critical in our arguments.

The content of this paper is organized as follows. In Sect. 2, we prove some basic properties of Brownian motion with measure-valued drift in arbitrary bounded open sets. In Sect. 3, we use the estimates of [21] to prove that the harmonic measures with respect to $X$ are uniformly (under scaling and translation) comparable to the corresponding harmonic measures with respect to Brownian motion if the domain $D$ is shrunk small enough. In Sect. 4, we prove a scale invariant boundary Harnack principle. Section 5 deals with Martin boundary and the Martin representation. We prove that the Martin boundary and the minimal Martin boundary of killed Brownian motion with measure-valued drift can all be identified with the Euclidean boundary if the domain is bounded and Lipschitz. In the final section we briefly indicate that all the results remain valid for diffusions with measure-valued drifts, that is, when $\Delta$ is replaced by a uniformly elliptic divergence form operator $\sum_{i, j=1}^{d} \partial_{i}\left(a_{i j} \partial_{j}\right)$ with $C^{1}$ coefficients or a uniformly elliptic non-divergence form operator $\sum_{i, j=1}^{d} a_{i j} \partial_{i} \partial_{j}$ with $C^{1}$ coefficients.

In this paper, we will use the following convention: the values of the constants $M_{1}, M_{2}, \ldots$ will remain the same throughout this paper, while the values of the 
constants $C_{1}, C_{2}, \ldots$ might change from one appearance to another. The labeling of the constants $C_{1}, C_{2}, \ldots$ starts anew in the statement of each result. In this paper, we use ":=" to denote a definition, which is read as "is defined to be".

\section{Some properties of Brownian motions with measure-valued drifts in bounded domains}

Throughout this paper we assume that $\mu=\left(\mu^{1}, \ldots, \mu^{d}\right)$ is fixed with each $\mu^{i}$ being a signed measure on $\mathbf{R}^{d}$ belonging to $\mathbf{K}_{d, 1}$ and that $X$ is a Brownian motion with drift $\mu$. In this section, we will study some basic properties of $X$ which will be needed later.

We know from (1.1) that there exist $c_{1}, c_{2}$ and $c_{3}$ such that for every positive $t_{0}$ and $\delta$,

$$
\begin{aligned}
\sup _{t \leq t_{0},|x-y|>\delta} q(t, x, y) & \leq c_{1} e^{c_{2} t_{0}} \sup _{t \leq t_{0},|x-y|>\delta} t^{-\frac{d}{2}} e^{-c_{3} \frac{|x-y|^{2}}{t}} \\
& \leq c_{1} e^{c_{2} t_{0}} \sup _{t \leq t_{0}} t^{-\frac{d}{2}} e^{-c_{3} \frac{\delta^{2}}{t}}<\infty
\end{aligned}
$$

Since

$$
\lim _{t_{0} \downarrow 0} \sup _{t \leq t_{0}} t^{-\frac{d}{2}} e^{-c_{3} \frac{\delta^{2}}{t}}=0,
$$

we have

$$
\lim _{t_{0} \downarrow 0} \sup _{t<t_{0},|x-y|>\delta} q(t, x, y)=0 .
$$

Moreover, using (2.1), we get

$$
\begin{gathered}
\sup _{t \leq t_{0}, x \in \mathbf{R}^{d}} \mathbf{P}_{x}\left(\left|X_{t}-x\right| \geq \delta\right) \leq c_{1} e^{c_{2} t_{0}} \sup _{t \leq t_{0}, x \in \mathbf{R}^{d}} \int_{|x-y| \geq \delta} t^{-\frac{d}{2}} e^{-c_{3} \frac{|x-y|^{2}}{t}} d y \\
=c_{4} e^{c_{2} t_{0}} \sup _{t \leq t_{0}} \int_{\delta}^{\infty} t^{-\frac{d}{2}} r^{d-1} e^{-c_{3} \frac{r^{2}}{t}} d r \leq c_{5} e^{c_{2} t_{0}} \int_{\frac{\delta}{\sqrt{t_{0}}}}^{\infty} u^{d-1} e^{-c_{3} u^{2}} d u
\end{gathered}
$$

for some $c_{4}=c_{4}(d)$ and $c_{5}=c_{5}(d)$. Thus

$$
\lim _{t_{0} \downarrow 0} \sup _{t \leq t_{0}, x \in \mathbf{R}^{d}} \mathbf{P}_{x}\left(\left|X_{t}-x\right| \geq \delta\right)=\lim _{t_{0} \downarrow 0} \sup _{t \leq t_{0}, x \in \mathbf{R}^{d}} \mathbf{P}_{x}\left(X_{t} \in \mathbf{R}^{d} \backslash B(x, \delta)\right)=0 .
$$

For any open set $D$, we use $\tau_{D}$ to denote the first exit time of $D$, i. e., $\tau_{D}=\inf \{t>$ 0 : $X_{t} \notin D$ \}. Using (2.3) we can easily prove the next lemma. 
Lemma 2.1 For any $\delta>0$, we have

$$
\lim _{s \downarrow 0} \sup _{x \in \mathbf{R}^{d}} \mathbf{P}_{x}\left(\tau_{B(x, \delta)} \leq s\right)=0 .
$$

Proof For any $t>0$ and any Borel set $A$ in $\mathbf{R}^{d}$, we put $N_{t}(x, A)=\mathbf{P}_{x}\left(X_{t} \in A\right)$. Then by an extended version of the strong Markov property (see [7, pp. 43-44]), we have for every $x \in \mathbf{R}^{d}$,

$$
\begin{aligned}
& \mathbf{P}_{x}\left(\tau_{B(x, \delta)} \leq s\right) \leq \mathbf{P}_{x}\left(\tau_{B(x, \delta)} \leq s, X_{s} \in B\left(x, \frac{\delta}{2}\right)\right)+\mathbf{P}_{x}\left(X_{s} \in B\left(x, \frac{\delta}{2}\right)^{c}\right) \\
& \leq \mathbf{E}_{x}\left[N_{s-\tau_{B(x, \delta)}}\left(X_{\tau_{B(x, \delta)}}, B\left(x, \frac{\delta}{2}\right)^{c}\right) ; \tau_{B(x, \delta)} \leq s\right]+\mathbf{P}_{x}\left(X_{s} \in B\left(x, \frac{\delta}{2}\right)^{c}\right)
\end{aligned}
$$

Now the conclusion of the lemma follows from (2.3).

Recall that, a point $z$ on the boundary $\partial D$ of an open set $D$ is said to be a regular boundary point if $\mathbf{P}_{x}\left(\tau_{D}=0\right)=1$. An open set $D$ is said to be regular if every point in $\partial D$ is a regular boundary point.

Proposition 2.2 Suppose $D$ is an open subset of $\mathbf{R}^{d}$ and $z \in \partial D$. If there is a cone $A$ with vertex $z$ such that $A \cap B(z, r) \subset D^{c}$ for some $r>0$, then $z$ is a regular boundary point of $D$.

Proof Without loss of generality, we may assume that $z=0$. For $n \geq 1$, put $r_{n}=r / n$. Under $\mathbf{P}_{0}$, we have

$$
\bigcap_{m=1}^{\infty} \bigcup_{n=m}^{\infty}\left\{X_{r_{n}} \in A \cap B(0, r)\right\} \subset\left\{\tau_{D}=0\right\}
$$

Hence

$$
\begin{aligned}
\mathbf{P}_{0}\left(\tau_{D}=0\right) & \geq \mathbf{P}_{0}\left(\bigcap_{m=1}^{\infty} \bigcup_{n=m}^{\infty}\left\{X_{r_{n}} \in A \cap B(0, r)\right\}\right) \\
& \geq \limsup _{n \rightarrow \infty} \mathbf{P}_{0}\left(X_{r_{n}} \in A \cap B(0, r)\right) \\
& \geq \limsup _{n \rightarrow \infty} c_{1} \int_{A \cap B(0, r)} r_{n}^{-\frac{d}{2}} e^{-\frac{c_{2}|x|^{2}}{r_{n}}} d x \\
& \geq \limsup _{n \rightarrow \infty} c_{1} \int_{A \cap B(0, n)} e^{-c_{2}|y|^{2}} d y>0 .
\end{aligned}
$$

The assertion of the proposition now follows from Blumenthal's zero-one law (Proposition I.5.17 in [7]). 
This result implies that all bounded Lipschitz domains, and in particular, all bounded $C^{1,1}$ domains, are regular. Repeating the argument in the second part of the proof of Theorem 1.23 in [11], we immediately get the following result.

Proposition 2.3 Suppose that $D$ is a domain in $\mathbf{R}^{d}$ and $f$ is a bounded Borel function on $\partial D$. If $z$ is a regular boundary point of $D$ and $f$ is continuous at $z$, then

$$
\lim _{\bar{D} \ni x \rightarrow z} \mathbf{E}_{x}\left[f\left(X_{\tau_{D}}\right) ; \tau_{D}<\infty\right]=f(z) .
$$

Proof We omit the details.

Given an open set $D \subset \mathbf{R}^{d}$, we define $X_{t}^{D}(\omega)=X_{t}(\omega)$ if $t<\tau_{D}(\omega)$ and $X_{t}^{D}(\omega)=$ $\partial$ if $t \geq \tau_{D}(\omega)$, where $\partial$ is a cemetery state. The process $X^{D}$ is called a killed Brownian motion with drift $\mu$ in $D$. Throughout this paper, we use the convention $f(\partial)=0$.

Let

$$
\begin{aligned}
k^{D}(t, x, y) & :=\mathbf{E}_{x}\left[q\left(t-\tau_{D}, X_{\tau_{D}}, y\right): \tau_{D}<t\right] \\
\text { and } q^{D}(t, x, y) & :=q(t, x, y)-k^{D}(t, x, y) .
\end{aligned}
$$

Then $q^{D}(t, x, y)$ is the transition density of $X^{D}$ i.e., for every $t>0$ and Borel set $A$,

$$
\mathbf{P}_{x}\left(X_{t}^{D} \in A\right)=\int_{A} q^{D}(t, x, y) d y .
$$

For the proof of (2.4), see [3, pp. 121-122] where only the strong Markov property was used.

Using some standard arguments (for example, $[3,11]$ ), we can show the following.

Theorem 2.4 For every $t>0, q^{D}(t, x, y)$ is jointly continuous on $D \times D$. For every $t>0$ and $s>0$,

$$
q^{D}(t+s, x, y)=\int_{D} q^{D}(t, x, z) q^{D}(s, z, y) d z .
$$

For every $(t, x, y) \in(0, \infty) \times D \times D, q^{D}(t, x, y)$ is strictly positive. If $z$ is a regular boundary point of $D$, then for any $t>0$ and $y \in D$,

$$
\lim _{D \ni x \rightarrow z} q^{D}(t, x, y)=0 .
$$

Proof First, we show that $k^{D}(t, x, \cdot)$ is continuous in $D$ for each $(t, x) \in(0, \infty) \times D$. Suppose $\left(t_{0}, x_{0}, y_{0}\right) \in(0, \infty) \times D \times D$ and $y_{n}$ converges to $y_{0}$ in D. Let $\delta_{0}:=$ $\operatorname{dist}\left(y_{0}, \partial D\right)$. Choose $n_{0}$ large such that $\operatorname{dist}\left(y_{n}, \partial D\right)>\frac{1}{2} \delta_{0}$ for every $n \geq n_{0}$. Given $\varepsilon>0$, using (2.2), we can choose $s>0$ small such that

$$
\sup _{n \geq n_{0}} \mathbf{E}_{x_{0}}\left[q\left(t_{0}-\tau_{D}, X_{\tau_{D}}, y_{n}\right): t_{0}-s<\tau_{D}<t_{0}\right]<\frac{\varepsilon}{2} .
$$


So using (2.1), the continuity of $q$ and the bounded convergence theorem, we get

$$
\lim _{n \rightarrow \infty} \mathbf{E}_{x_{0}}\left[q\left(t_{0}-\tau_{D}, X_{\tau_{D}}, y_{n}\right): t_{0}-s \geq \tau_{D}\right]=0
$$

Now assume $\left(t_{0}, x_{0}, y_{0}\right) \in(0, \infty) \times D \times D$ and $x_{n}$ converges to $x_{0}$ in $D$. Let

$$
h(s, x):=\mathbf{E}_{x}\left[\mathbf{E}_{X_{s}}\left[q\left(t_{0}-s-\tau_{D}, X_{\tau_{D}}, y_{0}\right): \tau_{D}<t_{0}-s\right]\right], \quad s<t_{0} .
$$

We know from (2.1) that $\mathbf{E}_{X_{s}}\left[q\left(t_{0}-s-\tau_{D}, X_{\tau_{D}}, y_{0}\right): \tau_{D}<t_{0}-s\right]$ is bounded. So by using the continuity of $q, h(s, \cdot)$ is continuous in $D$ for every $s<t_{0}$. Now using the Markov property, we have

$$
\begin{aligned}
& \left|k^{D}\left(t_{0}, x, y_{0}\right)-h(s, x)\right| \\
& =\mid \mathbf{E}_{x}\left[q\left(t_{0}-\tau_{D}, X_{\tau_{D}}, y_{0}\right): \tau_{D} \leq s\right] \\
& \quad+\mathbf{E}_{x}\left[\mathbf{E}_{X_{s}}\left[q\left(t_{0}-s-\tau_{D}, X_{\tau_{D}}, y_{0}\right): \tau_{D}<t_{0}-s\right] ; s<\tau_{D}<t\right]-h(s, x) \mid \\
& \quad \leq \mathbf{E}_{x}\left[q\left(t_{0}-\tau_{D}, X_{\tau_{D}}, y_{0}\right): \tau_{D} \leq s\right] \\
& \quad+\mathbf{E}_{x}\left[\mathbf{E}_{X_{s}}\left[q\left(t_{0}-s-\tau_{D}, X_{\tau_{D}}, y_{0}\right): \tau_{D}<t_{0}-s\right] ; \tau_{D} \leq s\right],
\end{aligned}
$$

which is less than

$$
2 c_{1} e^{c_{2} t_{0}}\left(\sup _{t \leq t_{0}} t^{\frac{d}{2}} e^{-c_{3} \frac{\delta_{0}^{2}}{t}}\right) \mathbf{P}_{x}\left(\tau_{D} \leq s\right) .
$$

By Lemma 2.1, the quantity above converges to zero uniformly on any compact subset $K$ of $D$ as $s \rightarrow 0$. Thus, given $\varepsilon$, by choosing $s_{0}$ small first and then choosing $n_{0}$ large, we get for $n \geq n_{0}$

$$
\begin{aligned}
& \left|k^{D}\left(t_{0}, x_{n}, y_{0}\right)-k^{D}\left(t_{0}, x_{0}, y_{0}\right)\right| \\
& \quad \leq 2 \sup _{m \geq 0}\left|k^{D}\left(t_{0}, x_{m}, y_{0}\right)-h\left(s_{0}, x_{m}\right)\right|+\left|h\left(s_{0}, x_{n}\right)-h\left(s_{0}, x_{0}\right)\right|<\varepsilon .
\end{aligned}
$$

Since $q(t, x, y)$ is continuous, we can now conclude that $q^{D}(t, x, y)$ is continuous in $x \in D$ and in $y \in D$. (2.5) can be proved using the Markov property and the continuity of $q^{D}(t, x, \cdot)$ (see [11, p. 35]).

We will prove the joint continuity of $q^{D}(t, \cdot, \cdot)$ by using (2.5) and (1.1). Assume $\left(t_{0}, x_{0}, y_{0}\right) \in(0, \infty) \times D \times D$ and $\left(x_{n}, y_{n}\right)$ converges to $\left(x_{0}, y_{0}\right)$ in $D \times D$. By (2.5), we have

$$
q^{D}\left(t_{0}, x_{n}, y_{n}\right)=\int_{D} q^{D}\left(\frac{1}{2} t_{0}, x_{n}, z\right) q^{D}\left(\frac{1}{2} t_{0}, z, y_{n}\right) d z
$$

It follows from (1.1) that $q^{D}\left(\frac{1}{2} t_{0}, x_{n}, z\right) q^{D}\left(\frac{1}{2} t_{0}, z, y_{n}\right)$ is bounded. So by the bounded convergence theorem, we have the joint continuity of $q^{D}(t, \cdot, \cdot)$. 
Finally, for $\left(t_{0}, x_{0}, y_{0}\right) \in(0, \infty) \times D \times D$, choose a smooth domain $G$ such that $x_{0}, y_{0} \in G \subset D$. Then $q^{D}\left(t_{0}, x_{0}, y_{0}\right) \geq q^{G}\left(t_{0}, x_{0}, y_{0}\right)>0$ by (1.4).

By Proposition 2.3, the last assertion of the theorem can be proved using the argument in the last paragraph of the proof of Theorem 2.4 of [11]. We omit the details.

In the remainder of this section we assume that $D$ is a bounded domain in $\mathbf{R}^{d}$. The next lemma is basically Lemma 6.1 in [21].

Lemma 2.5 There exist positive constants $C_{1}$ and $C_{2}$ depending on $D$ only via its diameter such that

$$
q^{D}(t, x, y) \leq C_{1} e^{-C_{2} t}, \quad(t, x, y) \in(1, \infty) \times D \times D .
$$

Proof With the help of (1.1), one can repeat the argument in the proof of Lemma 6.1 in [21] to arrive at the result with the constants depending on $D$ only via its diameter. We omit the details.

Combining the result above with (1.1) we know that there exist positive constants $C_{1}$ and $C_{2}$ depending on $D$ via its diameter such that for any $(t, x, y) \in(0, \infty) \times D \times D$,

$$
q^{D}(t, x, y) \leq C_{1} t^{-\frac{d}{2}} e^{-\frac{C_{2}|x-y|^{2}}{2 t}} .
$$

Therefore the Green function

$$
G_{D}(x, y):=\int_{0}^{\infty} q^{D}(t, x, y) d t
$$

is finite for $x \neq y$ and

$$
G_{D}(x, y) \leq C_{3} \frac{1}{|x-y|^{d-2}}
$$

for some $C_{3}=C_{3}(\operatorname{diam}(D))>0$.

Theorem 2.6 $G_{D}(x, y)$ is strictly positive and jointly continuous on $(D \times D) \backslash\{(x, y)$ : $x=y\} . G_{D}(x, y)$ is infinite if and only if $x=y$. For any $y \in D$,

$$
\lim _{x \rightarrow y} G_{D}(x, y)=\lim _{x \rightarrow y} G_{D}(y, x)=\infty
$$

Moreover, if $z$ is a regular boundary point of $D$, then for any $y \in D$,

$$
\lim _{D \ni x \rightarrow z} G_{D}(x, y)=0 .
$$

Proof Suppose $\left(x_{n}, y_{n}\right)$ converge to $\left(x_{0}, y_{0}\right)$ with $x_{0} \neq y_{0}$ and there exist disjoint compact sets $K_{1}$ and $K_{2}$ such that $x_{n} \in K_{1}$ and $y_{n} \in K_{2}$. Let $\delta:=\operatorname{dist}\left(K_{1}, K_{2}\right)$. Then 
by (2.7), there exist $C_{1}$ and $C_{2}$ depending on $D$ only via its diameter such that for any $t \in(0, \infty)$

$$
q^{D}\left(t, x_{n}, y_{n}\right) \leq C_{1} t^{-\frac{d}{2}} e^{-\frac{C_{2} \delta^{2}}{2 t}}=: f(t)
$$

Since $f(t)$ is in $L^{1}((0, \infty))$, the dominated convergence theorem and Theorem 2.4 give the joint continuity on $(D \times D) \backslash\{(x, y): x=y\}$. The strict positivity of $G_{D}(x, y)$ follows from the strict positivity of $q^{D}(t, x, y)$.

Fix $x_{0} \in D$ and choose a smooth domain $G$ such that $\overline{B\left(x_{0}, \frac{1}{2} \rho\left(x_{0}\right)\right)} \subset G \subset D$. Then by (1.3), there exists positive constants $c_{1}$ and $c_{2}$ such that

$$
\begin{aligned}
q^{D}\left(t, x_{0}, y\right) & \geq q^{G}\left(t, x_{0}, y\right) \geq c_{1}\left(1 \wedge \frac{\rho\left(x_{0}\right)^{2}}{t}\right) t^{-\frac{d}{2}} e^{-\frac{c_{2}\left|x_{0}-y\right|^{2}}{t}}, \\
t & <1 \text { and } y \in B\left(x_{0}, \frac{1}{4} \rho\left(x_{0}\right)\right) .
\end{aligned}
$$

So

$$
G_{D}\left(x_{0}, x_{0}\right) \geq c_{1} \int_{0}^{\rho\left(x_{0}\right)^{2} \wedge 1} t^{-\frac{d}{2}} d t=\infty .
$$

Also, by Fatou's lemma,

$$
\begin{aligned}
\liminf _{y \rightarrow x_{0}} G_{D}\left(x_{0}, y\right) & \geq \liminf _{y \rightarrow x_{0}} c_{1} \int_{0}^{\rho\left(x_{0}\right)^{2} \wedge 1} t^{-\frac{d}{2}} e^{-\frac{c_{2}\left|x_{0}-y\right|^{2}}{t}} d t \\
& \geq c_{1} \int_{0}^{\rho\left(x_{0}\right)^{2} \wedge 1} t^{-\frac{d}{2}} \liminf _{y \rightarrow x_{0}} e^{-\frac{c_{2}\left|x_{0}-y\right|^{2}}{t}} d t \\
& =c_{1} \int_{0}^{\rho\left(x_{0}\right)^{2} \wedge 1} t^{-\frac{d}{2}} d t=\infty .
\end{aligned}
$$

The other claim in (2.9) can be proved similarly.

The last assertion follows easily from the last assertion of Theorem 2.4.

Therefore $G_{D}$ satisfies the condition (i), (ii), (iii) and (vi) in [12] and hence the Riesz representation (see [12, p. 28] and [13, pp. 186-187]) holds. To make the statement above precise, we first recall some definitions.

Definition 2.7 Suppose that $U$ is an open subset of $\mathbf{R}^{d}$. A Borel function $u$ defined on $\mathbf{R}^{d}$ is said to be 
(1) harmonic in $U$ with respect to $X$ if

$$
u(x)=\mathbf{E}_{x}\left[u\left(X_{\tau_{B}}^{U}\right)\right], \quad x \in B
$$

for every bounded open set $B$ with $\bar{B} \subset U$;

(2) regular harmonic in $U$ with respect to $X$ if it is harmonic for $X^{U}$ and for each $x \in U$,

$$
u(x)=\mathbf{E}_{x}\left[u\left(X_{\tau_{U}}\right)\right]
$$

(3) superharmonic in $U$ with respect to $X$ if $u$ is non-negative and

$$
u(x) \geq \mathbf{E}_{x}\left[u\left(X_{\tau_{B}}^{U}\right)\right], \quad x \in B
$$

for every bounded open set $B$ with $\bar{B} \subset U$;

(4) excessive for $X^{U}$ if $u$ is non-negative and

$$
\begin{aligned}
& u(x) \geq \mathbf{E}_{x}\left[u\left(X_{t}^{U}\right)\right]=\mathbf{E}_{x}\left[u\left(X_{t}\right): \tau_{U}>t\right] \text { and } \\
& u(x)=\lim _{t \downarrow 0} \mathbf{E}_{x}\left[u\left(X_{t}^{U}\right)\right], \quad t>0, x \in U,
\end{aligned}
$$

(5) a potential for $X^{U}$ if it is excessive for $X^{U}$ and for every sequence $\left\{U_{n}\right\}_{n \geq 1}$ of open sets with $\overline{U_{n}} \subset U_{n+1}$ and $\cup_{n} U_{n}=U$,

$$
\lim _{n \rightarrow \infty} \mathbf{E}_{x}\left[u\left(X_{\tau_{U_{n}}}^{U}\right)\right]=0, \quad \text { for every } x \in U \text { with } u(x)<\infty
$$

It is well known that $u$ is excessive for $X^{U}$ if and only if $f$ is lower-semicontinuous in $U$ and superharmonic in $U$ with respect to $X$. (See Theorem 4.5.3 in [10] for the Brownian motion case, and the proof there can adapted easily to the present case.)

Harmonic functions with respect to $X$ are continuous if they are locally bounded.

Proposition 2.8 Assume that $U$ is an open subset of $\mathbf{R}^{d}$. Then any locally bounded harmonic function u in $U$ is continuous. In particular, for any bounded Borel function $f$ in $\partial U, \mathbf{E}_{x}\left[f\left(X_{\tau_{U}}\right)\right]$ is continuous and regular harmonic in $U$.

Proof Fix $x \in U$ and choose smooth compact open subsets $D_{1}$ and $D_{2}$ with $x \in D_{1} \subset$ $\overline{D_{1}} \subset D_{2} \subset \overline{D_{2}} \subset U$. Let $L:=\|u\|_{L^{\infty}\left(\overline{D_{2}}\right)}<\infty$. We assume that $\left\{x_{n}\right\}_{n \geq 1} \subset D_{1}$ and 
$x_{n} \rightarrow x$. By (2.10) and the Markov property,

$$
\begin{aligned}
& \left|u\left(x_{n}\right)-u(x)\right|=\left|\mathbf{E}_{x_{n}}\left[u\left(X_{\tau_{D_{2}}}\right)\right]-\mathbf{E}_{x}\left[u\left(X_{\tau_{D_{2}}}\right)\right]\right| \\
& \leq 2 L \sup _{z \in D_{1}} \mathbf{P}_{z}\left(\tau_{D_{2}} \leq t\right)+\mid \mathbf{E}_{x_{n}}\left[\mathbf{E}_{X_{t}}\left[u\left(X_{\tau_{D_{2}}}\right)\right]: \tau_{D_{2}}>t\right] \\
& \quad-\mathbf{E}_{x}\left[\mathbf{E}_{X_{t}}\left[u\left(X_{\tau_{D_{2}}}\right)\right]: \tau_{D_{2}}>t\right] \mid \\
& =2 L \sup _{z \in D_{1}} \mathbf{P}_{z}\left(\tau_{D_{2}} \leq t\right)+\left|\mathbf{E}_{x_{n}}\left[\mathbf{E}_{X_{t}^{D_{2}}}\left[u\left(X_{\tau_{D_{2}}}\right)\right]\right]-\mathbf{E}_{x}\left[\mathbf{E}_{X_{t}^{D_{2}}}\left[u\left(X_{\tau_{D_{2}}}\right)\right]\right]\right| \\
& \leq 2 L \sup _{z \in D_{1}} \mathbf{P}_{z}\left(\tau_{D_{2}} \leq t\right)+L \int q_{D_{2}}\left|q^{D_{2}}\left(t, x_{n}, y\right)-q^{D_{2}}(t, x, y)\right| d y
\end{aligned}
$$

Given $\varepsilon>0$, by Lemma 2.1, there exists $t_{0}>0$ such that

$$
\sup _{z \in D_{1}} \mathbf{P}_{z}\left(\tau_{D_{2}} \leq t_{0}\right)<\frac{\varepsilon}{8 L}
$$

By (1.3),

$$
\begin{aligned}
& \int_{D_{2}}\left|q^{D_{2}}\left(t_{0}, x_{n}, y\right)-q^{D_{2}}\left(t_{0}, x, y\right)\right| d y \\
& =\left(\int_{D_{2} \cap\left\{\operatorname{dist}\left(y, \partial D_{2}\right)<\delta\right\}}+\int_{D_{2} \backslash\left\{\operatorname{dist}\left(y, \partial D_{2}\right) \geq \delta\right\}}\right)\left|q^{D_{2}}\left(t_{0}, x_{n}, y\right)-q^{D_{2}}\left(t_{0}, x, y\right)\right| d y \\
& \leq c_{1}\left|D_{2}\right| \delta t_{0}^{-\frac{d+1}{2}}+\underset{D_{2} \cap\left\{\operatorname{dist}\left(y, \partial D_{2}\right) \geq \delta\right\}}{\int_{D^{2}}^{D_{2}}\left(t_{0}, x_{n}, y\right)-q^{D_{2}}\left(t_{0}, x, y\right) \mid d y,}
\end{aligned}
$$

for some $c_{1}$. Now we choose $\delta$ small so that $c_{1}\left|D_{2}\right| L \delta t_{0}^{-\frac{d+1}{2}}<\frac{\varepsilon}{4}$. The convergence of the second term in the last equation above follows from the uniform continuity of $q^{D_{2}}\left(t_{0}, \cdot, \cdot\right)$ on $\overline{D_{1}} \times\left(D_{2} \cap\left\{\operatorname{dist}\left(y, \partial D_{2}\right) \geq \delta\right\}\right)$ (see Theorem 3.1 in [21]). Thus we have proved the Proposition.

Now we state some properties of Green functions. Since $X^{D}$ is a transient diffusion, it satisfies the conditions in [23]. Thus, combining Theorem 1 in [23], the results in [12, p. 28], Corollary 2 to Theorem 2, Proposition 11 and Theorems 5-6 in [13], we have

Theorem 2.9 (1) For each $y, x \rightarrow G_{D}(x, y)$ excessive for $X^{D}$. Moreover, for every open subset $U$ of $D$, we have

$$
\mathbf{E}_{x}\left[G_{D}\left(X_{T_{U}}^{D}, y\right)\right]=G_{D}(x, y), \quad(x, y) \in D \times U
$$


where $T_{U}:=\inf \left\{t>0: X_{t}^{D} \in U\right\}$. In particular, for every $y \in D$ and $\varepsilon>0$, $G_{D}(\cdot, y)$ is regular harmonic in $D \backslash B(y, \varepsilon)$ with respect to $X^{D}$.

(2) For every Radon measure $v$ on $D$,

$$
G_{D} v(x):=\int_{D} G_{D}(x, y) v(d y)
$$

is a potential for $X^{D}$. Conversely, if $u$ is a potential for $X^{D}$, then there exists a unique Radon measure $v$ on $D$ such that $u=G_{D}$.

(3) If $f$ is an excessive function for $X^{D}$ which is not identically zero, then there exists a unique Radon measure $v$ on $D$ and a harmonic function $h$ for $X^{D}$ such that $f=G_{D} v+h$.

The proof of the next proposition can be found in the proofs of Theorems 2-3 in [23]. We put the proof here for reader's convenience.

Proposition 2.10 If $h$ is a nonnegative harmonic for $X^{D}$ and $U$ is an open subset of $D$ with $\bar{U} \subset D$, then there exists a Radon measure v supported on $\partial U$ such that $h=G_{D} v$ in $U$. In particular, every nonnegative harmonic function for $X^{D}$ is continuous.

Proof Note that from Proposition 2.8, we know that every bounded harmonic function is continuous. Since $h$ is the increasing limit of $\mathbf{E}_{x}\left[h\left(X_{\tau_{V}}^{D}\right) \wedge k\right]$ for every open subset $V$ of $D$ with $\bar{V} \subset D, h$ is lower semicontinuous. Thus $h$ is excessive. Let $T_{U}:=$ $\inf \left\{t>0: X_{t}^{D} \in U\right\}$. Since $h$ is excessive, Corollary 1 to Theorem 2 in [13] implies that there exists a Radon measure $v$ supported on $\bar{U}$ such that $\mathbf{E}_{x}\left[h\left(X_{T_{U}}^{D}\right)\right]=G_{D} v(x)$ for all $x \in D$. Since

$$
G_{D} v(x)=\int_{U} G_{D}(x, y) v(d y)+\int_{\partial U} G_{D}(x, y) v(d y)=: h_{1}(x)+h_{2}(x), \quad x \in D
$$

and $h_{1}$ and $h_{2}$ are excessive (Theorem 2.9), $h_{1}$ and $h_{2}$ must be harmonic with respect to $X$ in $U$. Let $K$ be a compact subset of $U$. By the harmonicity of $h_{1}$, we have

$$
\mathbf{E}_{x}\left[h_{1}\left(X_{T_{K^{c}}}^{D}\right)\right]=\int_{\partial U} G_{D}(x, y) v(d y) .
$$

But, by Corollary 1 to Theorem 2 in [13], $v$ can not charge the interior of $K$. Since $K$ is an arbitrary compact subset of $U$, we get that $h_{1}$ is identically zero and $v$ is supported by $\partial U$. Therefore we have shown $h(x)=\mathbf{E}_{x}\left[h\left(X_{T_{U}}^{D}\right)\right]=G_{D} v(x)$ for $x \in U$. Now the continuity of $h$ follows from the continuity of $G_{D}$.

\section{Green function estimates and comparison of harmonic measures}

In this section, we assume that $D$ is a bounded $C^{1,1}$ domain. Recall that a bounded domain $D$ in $\mathbf{R}^{d}$ is said to be a $C^{1,1}$ domain if there is a localization radius $r_{0}>0$ 
and a constant $\Lambda>0$ such that for every $Q \in \partial D$, there is a $C^{1,1}$ function $\phi=\phi_{Q}$ : $\mathbf{R}^{d-1} \rightarrow \mathbf{R}$ satisfying $\phi(0)=|\nabla \phi(0)|=0,\|\nabla \phi\|_{\infty} \leq \Lambda,|\nabla \phi(x)-\nabla \phi(z)| \leq$ $\Lambda|x-z|$, and an orthonormal coordinate system $C S_{Q}$ with origin at $Q$ such that

$$
\begin{aligned}
B\left(Q, r_{0}\right) \cap D & =B\left(Q, r_{0}\right) \cap\left\{y=\left(y_{1}, \ldots, y_{d-1}, y_{d}\right)\right. \\
& \left.:=\left(\tilde{y}, y_{n}\right) \text { in } C S_{Q}: y_{d}>\phi(\tilde{y})\right\} .
\end{aligned}
$$

The pair $\left(r_{0}, \Lambda\right)$ is called the characteristics of the $C^{1,1}$ domain $D$. The main objective of this section is to show that the harmonic measures with respect to $X$ are uniformly (under scale and translation) comparable to the corresponding harmonic measures with respect to Brownian motion if the domain $D$ is shrunk small enough.

Let $p^{D}(t, x, y)$ be the density of Brownian motion killed upon exiting $D$. It is known that for any $T>0$, there exist positive constants $M_{i}, i=1, \ldots, 5$, depending on $T$ and $D$ such that

$$
M_{1} \psi_{D}(t, x, y) t^{-\frac{d}{2}} e^{-\frac{M_{2}|x-y|^{2}}{t}} \leq p^{D}(t, x, y) \leq M_{3} \psi_{D}(t, x, y) t^{-\frac{d}{2}} e^{-\frac{M_{4}|x-y|^{2}}{t}}
$$

and

$$
\left|\nabla_{x} p^{D}(t, x, y)\right| \leq M_{5} t^{-\frac{d+1}{2}} e^{-\frac{M_{4}|x-y|^{2}}{t}}
$$

for all $(t, x, y) \in(0, T] \times D \times D$. (3.1) was proved in [15,28] and (3.2) was proved in [17]. By taking $M_{4}$ smaller if necessary, we can assume that $M_{4} \leq 1 / 2$. Differentiating with respect to $x$ in the equation

$$
p^{D}(t, x, y)=\int_{D} p^{D}\left(\frac{t}{2}, x, z\right) p^{D}\left(\frac{t}{2}, z, y\right) d z
$$

and using the above estimates on $p^{D}(t, x, y)$ and $\nabla_{x} p^{D}(t, x, y)$ we get

$$
\begin{aligned}
\left|\nabla_{x} p^{D}(t, x, y)\right| & \leq 2^{d+1} M_{3} M_{5} \int_{D} t^{-\frac{d+1}{2}} e^{-\frac{2 M_{4}|x-z|^{2}}{t}} \rho(y) t^{-\frac{d+1}{2}} e^{-\frac{2 M_{4}|z-y|^{2}}{t}} d z \\
& \leq 2^{d+1} M_{3} M_{5} \rho(y) \int_{\mathbf{R}^{d}} t^{-\frac{d+1}{2}} e^{-\frac{2 M_{4}|x-z|^{2}}{t}} t^{-\frac{d+1}{2}} e^{-\frac{2 M_{4}|z-y|^{2}}{t}} d z \\
& :=M_{6} \rho(y) t^{-\frac{d+2}{2}} e^{-\frac{M 4|x-y|^{2}}{t}} .
\end{aligned}
$$

In the last equality above, we have used the semigroup property of the Gaussian kernel. Combining this with (3.2) we see that, for any $T>0$, there exists a positive constant $M_{7}$ such that

$$
\left|\nabla_{x} p^{D}(t, x, y)\right| \leq M_{7}\left(1 \wedge \frac{\rho(y)}{\sqrt{t}}\right) t^{-\frac{d+1}{2}} e^{-\frac{M_{4}|x-y|^{2}}{t}}
$$


for all $(t, x, y) \in(0, T] \times D \times D$. Since $p^{D}(t, x, y) \leq p(t, x, y)$, there exists a positive constant $M_{8}$ depending only on $d$ such that

$$
p^{D}(t, x, y) \leq M_{8} t^{-\frac{d}{2}} e^{-M_{4} \frac{|x-y|^{2}}{t}}, \quad(t, x, y) \in(0, \infty) \times D \times D .
$$

By combining this with (3.2), Lemma 2.5 (works for $p^{D}$ ) and the semigroup property, we can easily get that there exists positive constant $M_{9}$ depending on $D$ such that

$$
\left|\nabla_{x} p^{D}(t, x, y)\right| \leq M_{9} t^{-\frac{d+1}{2}} e^{-M_{4} \frac{|x-y|^{2}}{t}}, \quad(t, x, y) \in(0, \infty) \times D \times D .
$$

For any $z \in \mathbf{R}^{d}$ and $r \in(0,1)$, put $D_{r}^{z}:=z+r D$ and let $\rho_{D_{r}^{z}}(x)$ be the distance between $x$ and $\partial D_{r}^{z}$. By the translation and scaling property of $p^{D}$, we see that the constants in (3.4)-(3.5) are invariant under translation and Brownian scaling. Thus we have

$$
p^{D_{r}^{z}}(t, x, y) \leq M_{8} t^{-\frac{d}{2}} e^{-M_{4} \frac{|x-y|^{2}}{t}}, \quad(t, x, y) \in(0, \infty) \times D_{r}^{z} \times D_{r}^{z}
$$

and

$$
\left|\nabla_{x} p^{D_{r}^{z}}(t, x, y)\right| \leq M_{9} t^{-\frac{d+1}{2}} e^{-M_{4} \frac{|x-y|^{2}}{t}}, \quad(t, x, y) \in(0, \infty) \times D_{r}^{z} \times D_{r}^{z} .
$$

We will suppress indices above from $D_{r}^{z}$ when it is clear from the context.

By taking scale and translation invariance into consideration in the proof of Theorem 4.2 of [21], we have observed at the beginning of Sect. 5 of [21] that for any $T>0$, there exist positive constants $t_{0}$ and $M_{j}, 10 \leq j \leq 14$, independent of $z$ and $r$ and depending on $\mu$ only via the rate at which $\max _{1 \leq i \leq d} M_{\mu^{i}}^{1}(r)$ goes to zero, such that

$$
M_{10} t^{-\frac{d}{2}} \psi_{D_{r}^{z}}(t, x, y) e^{-\frac{M_{11}|x-y|^{2}}{2 t}} \leq q^{D_{r}^{z}}(t, x, y) \leq M_{12} t^{-\frac{d}{2}} \psi_{D_{r}^{z}}(t, x, y) e^{-\frac{M_{13}|x-y|^{2}}{2 t}}
$$

and

$$
\left|\nabla_{x} q^{D_{r}^{z}}(t, x, y)\right| \leq M_{14}\left(1 \wedge \frac{\rho_{D_{r}^{z}}(y)}{\sqrt{t}}\right) t^{-\frac{d+1}{2}} e^{-\frac{M_{13}|x-y|^{2}}{2 t}}
$$

for all $(t, x, y) \in\left(0, t_{0} \wedge\left(r^{2} T\right)\right] \times D_{r}^{z} \times D_{r}^{z}$. We now show the scale and translation invariant version of the Green function estimate of $X^{D}$ when $D$ is a bounded $C^{1,1}$ domain.

First we show the lower bound of $G_{D_{r}^{z}}$, which is a direct consequence of the lower estimates of $q^{D_{r}^{z}}$ in (3.8). In the next theorem we will take $T=\operatorname{diam}(D)^{2}$, and let $t_{0}$ be the corresponding constant in (3.8). Recall from [21] that

$$
M_{\nu}(r):=\sup _{x \in \mathbf{R}^{d}} \int_{|x-y| \leq r} \frac{|v|(d y)}{|x-y|^{d-1}}, \quad N_{\nu}(t):=\sup _{x \in \mathbf{R}^{d}} \int_{0}^{t} \int_{\mathbf{R}^{d}} s^{-\frac{1}{2}} p(s, x, y)|v|(d y) d s .
$$


Theorem 3.1 Let $D$ be a bounded $C^{1,1}$ domain in $\mathbf{R}^{d}$. There exists a constant $C_{1}=$ $C_{1}(D, \mu)>0$ depending on $\mu$ only via the rate at which $\max _{1 \leq i \leq d} M_{\mu^{i}}(r)$ goes to zero such that for $r \leq \frac{\sqrt{t}_{0}}{\operatorname{diam}(D)}, z \in \mathbf{R}^{d}$ and $x, y \in D_{r}^{z}$,

$$
G_{D_{r}^{z}}(x, y) \geq C_{1}\left(1 \wedge \frac{\rho_{D_{r}^{z}}(x)}{|x-y|}\right)\left(1 \wedge \frac{\rho_{D_{r}^{z}}(y)}{|x-y|}\right) \frac{1}{|x-y|^{d-2}} .
$$

Proof It is easy to see that $\operatorname{diam}\left(D_{r}^{z}\right)^{2}=r^{2} \operatorname{diam}(D)^{2}$. For $r \leq \frac{\sqrt{t}_{0}}{\operatorname{diam}(D)}$, by (3.8), we have

$$
\begin{aligned}
G_{D_{r}^{z}}(x, y) & \geq \int_{0}^{\operatorname{diam}\left(D_{r}^{z}\right)^{2}} q^{D_{r}^{z}}(t, x, y) d t \\
& \geq M_{10} \int_{0}^{\operatorname{diam}\left(D_{r}^{z}\right)^{2}}\left(1 \wedge \frac{\rho_{D_{r}^{z}}(x)}{\sqrt{t}}\right)\left(1 \wedge \frac{\rho_{D_{r}^{z}}(y)}{\sqrt{t}}\right) t^{-\frac{d}{2}} e^{-\frac{M_{11}|x-y|^{2}}{2 t}} d t \\
& \geq \frac{M_{10}}{|x-y|^{d-2}} \int_{1}^{\infty} u^{\frac{d-4}{2}}\left(1 \wedge \frac{\sqrt{u} \rho_{D_{r}^{z}}(x)}{|x-y|}\right)\left(1 \wedge \frac{\sqrt{u} \rho_{D_{r}^{z}}(y)}{|x-y|}\right) e^{-\frac{1}{2} M_{11} u} d u \\
& \geq C_{1}\left(1 \wedge \frac{\rho_{D_{r}^{z}}(x)}{|x-y|}\right)\left(1 \wedge \frac{\rho_{D_{r}^{z}}(y)}{|x-y|}\right) \frac{1}{|x-y|^{d-2}}
\end{aligned}
$$

where $C_{1}=M_{10} \int_{1}^{\infty} u^{\frac{d-4}{2}} e^{-\frac{1}{2} M_{11} u} d u$

The following lemmas will be needed in proving the upper bound of the Green functions.

Lemma 3.2 For any $a, r>0, x_{0} \in \mathbf{R}^{d}$ and measure $\mu$ on $\mathbf{R}^{d}$, there exists constant $C_{1}=C_{1}(a, d)$ such that

$$
\sup _{u \in B\left(x_{0}, r\right)} \int_{0}^{\infty} \int_{B\left(x_{0}, r\right)} s^{-\frac{d+1}{2}} e^{-\frac{a|u-z|^{2}}{4 s}} \mu(d z) d s \leq C_{1}(a, d) N_{\mu}\left(\frac{r^{2}}{a}\right) .
$$

Proof Fix $a, r>0, x_{0} \in \mathbf{R}^{d}$ and a measure $\mu$ on $\mathbf{R}^{d}$. Since

$$
\sup _{u \in B\left(x_{0}, r\right)} \int_{0}^{\frac{r^{2}}{2}} \int_{B\left(x_{0}, r\right)} s^{-\frac{d+1}{2}} e^{-\frac{a|u-z|^{2}}{4 s}} \mu(d z) d s \leq\left(\frac{2}{a}\right)^{\frac{d-1}{2}}(2 \pi)^{\frac{d}{2}} N_{\mu}\left(\frac{r^{2}}{a}\right)
$$


we only need to show that

$$
\sup _{u \in B\left(x_{0}, r\right)} \int_{\frac{r^{2}}{2}}^{\infty} \int_{B\left(x_{0}, r\right)} s^{-\frac{d+1}{2}} e^{-\frac{a|u-z|^{2}}{4 s}} \mu(d z) d s \leq c(a, d) N_{\mu}\left(\frac{r^{2}}{a}\right) .
$$

Since $\left\{z:\left|x_{0}-z\right|<r\right\} \subset\{z:|u-z|<2 r\}$ for every $u \in B\left(x_{0}, r\right)$, we have

$$
\begin{aligned}
& \sup _{u \in B\left(x_{0}, r\right)} \int_{\frac{r^{2}}{2}}^{\infty} \int_{B\left(x_{0}, r\right)} s^{-\frac{d+1}{2}} e^{-\frac{a|u-z|^{2}}{4 s}} \mu(d z) d s \\
& \leq \sup _{u \in B\left(x_{0}, r\right)} \int_{\frac{r^{2}}{2}}^{\infty} \int_{|u-z|<2 r} s^{-\frac{d+1}{2}} e^{-\frac{a|u-z|^{2}}{4 s}} \mu(d z) d s \\
& \leq \int_{\frac{r^{2}}{2}}^{\infty} \sup _{u \in B\left(x_{0}, r\right)} \int_{|u-z|<2 r} s^{-\frac{d+1}{2}} e^{-\frac{a|u-z|^{2}}{4 s}} \mu(d z) d s \leq\left(\int_{\frac{r^{2}}{2}}^{\infty} s^{-\frac{d+1}{2}} d s\right)(2 r)^{d-1} M_{\mu}(2 r)
\end{aligned}
$$

In the last inequality above, we used the fact that for any signed measure $v$,

$$
\sup _{x \in \mathbf{R}^{d}}|\nu|(\overline{B(x, r)}) \leq r^{d-1} M_{\mu}(r)
$$

(see (2.1) in [21]). Using the change of variable $l=\frac{r^{2}}{s}$, we have

$$
\int_{\frac{r^{2}}{2}}^{\infty} s^{-\frac{d+1}{2}} d s=r^{-d+1} \int_{0}^{2} l^{\frac{d-3}{2}} d l
$$

On the other hand, we have

$$
\begin{aligned}
& \int_{0}^{\frac{r^{2}}{a}} \int_{\mathbf{R}^{d}} s^{-\frac{1}{2}} p(s, x, y) \mu(d y) d s=\int_{\mathbf{R}^{d}} \int_{0}^{\frac{r^{2}}{a}} s^{-\frac{1}{2}} p(s, x, y) d s \mu(d y) \\
& \geq \int_{|x-y| \leq 2 r} 2^{\frac{d-1}{2}}(2 \pi)^{-\frac{d}{2}}|x-y|^{-d+1} \int_{\frac{a|x-y|^{2}}{2 r^{2}}}^{\infty} u^{\frac{d-3}{2}} e^{-u} d u \mu(d y) \\
& \geq 2^{\frac{d-1}{2}}(2 \pi)^{-\frac{d}{2}}\left(\int_{2 a}^{\infty} u^{\frac{d-3}{2}} e^{-u} d u\right)_{|x-y| \leq 2 r}|x-y|^{-d+1} \mu(d y) .
\end{aligned}
$$


Thus

$$
M_{\mu}(2 r) \leq C_{0}(a, d) N_{\mu}\left(\frac{r^{2}}{a}\right)
$$

Therefore

$$
\left(\int_{\frac{r^{2}}{2}}^{\infty} s^{-\frac{d+1}{2}} d s\right)(2 r)^{d-1} M_{\mu}(2 r) \leq C_{1}(a, d) N_{\mu}\left(\frac{r^{2}}{a}\right) .
$$

The proof of the next lemma is almost identical to the proof of Lemma 3.1 of [27], so we skip the proof.

Lemma 3.3 For any $a>0$, there exist positive constants $C_{1}, C_{2}$ depending only on $a$ and $d$ such that for any measure $\mu$ on $\mathbf{R}^{d}$

$$
\begin{aligned}
& \int_{0}^{t} \int_{D} s^{-\frac{d}{2}} e^{-\frac{a|x-z|^{2}}{2 s}}(t-s)^{-\frac{d+1}{2}} e^{-\frac{a|z-y|^{2}}{t-s}} \mu(d z) d s \\
& \leq C_{1} t^{-\frac{d}{2}} e^{-\frac{a|x-y|^{2}}{2 t}} \sup _{u \in D} \int_{0}^{t} \int_{D} s^{-\frac{d+1}{2}} e^{-\frac{a|u-z|^{2}}{4 s}} \mu(d z) d s
\end{aligned}
$$

and

$$
\begin{aligned}
& \int_{0}^{t} \int_{D} s^{-\frac{d+1}{2}} e^{-\frac{a|x-z|^{2}}{2 s}}(t-s)^{-\frac{d+1}{2}} e^{-\frac{a|z-y|^{2}}{t-s}} \mu(d z) d s \\
& \leq C_{2} t^{-\frac{d+1}{2}} e^{-\frac{a|x-y|^{2}}{2 t}} \sup _{u \in D} \int_{0}^{t} \int_{D} s^{-\frac{d+1}{2}} e^{-\frac{a|u-z|^{2}}{4 s}} \mu(d z) d s
\end{aligned}
$$

for all $(t, x, y) \in(0, \infty) \times D \times D$.

Combining Lemmas 3.2 and 3.3, we have the following lemma.

Lemma 3.4 For any $a>0$, there exist positive constants $L_{3}, L_{4}$ depending only on $a$ and $d$ such that for any $r, t>0$, measure $\mu$ on $\mathbf{R}^{d}$ and an open subset $D$ with $\operatorname{diam}(D)<2 r$

$$
\int_{0}^{t} \int_{D} s^{-\frac{d}{2}} e^{-\frac{a|x-z|^{2}}{2 s}}(t-s)^{-\frac{d+1}{2}} e^{-\frac{a|z-y|^{2}}{t-s}} \mu(d z) d s \leq L_{3} N_{\mu}\left(\frac{r^{2}}{a}\right) t^{-\frac{d}{2}} e^{-\frac{a|x-y|^{2}}{2 t}}
$$


and

$$
\int_{0}^{t} \int_{D} s^{-\frac{d+1}{2}} e^{-\frac{a|x-z|^{2}}{2 s}}(t-s)^{-\frac{d+1}{2}} e^{-\frac{a|z-y|^{2}}{t-s}} \mu(d z) d s \leq L_{4} N_{\mu}\left(\frac{r^{2}}{a}\right) t^{-\frac{d+1}{2}} e^{-\frac{a|x-y|^{2}}{2 t}}
$$

Using Lemma 3.4 and the estimates (3.6)-(3.7), we can show the global upper estimate of $q^{D}$ if the diameter of $D$ is sufficiently small.

Theorem 3.5 There exists constant $r_{0}>0$ depending on $M_{4}, M_{8}$ and $M_{9}$ such that for any $r \leq r_{0}$ and $z \in \mathbf{R}^{d}$

$$
q^{D_{r}^{z}}(t, x, y) \leq 2 M_{8} t^{-\frac{d}{2}} e^{-\frac{M_{4}|x-y|^{2}}{2 t}}
$$

and

$$
\left|\nabla_{x} q^{D_{r}^{z}}(t, x, y)\right| \leq 2 M_{9} t^{-\frac{d+1}{2}} e^{-\frac{M_{4}|x-y|^{2}}{2 t}}
$$

for all $(t, x, y) \in(0, \infty) \times D_{r}^{z} \times D_{r}^{z}$.

Proof By Theorems 4.5 of [21], without loss of generality, we may assume that $\mu(d x)=U(x) d x$ where $U$ is smooth and bounded. By the translation and scaling property, we can also assume that $z=0$ and the diameter of $D$ is 2 , so that there exist $x_{1}$ such that $D \subset B\left(x_{1}, 1\right)$. Let $D_{r}=r D$ and $B_{r}=B\left(r x_{1}, r\right)$ so that $D_{r} \subset B_{r}$. By Theorem 6 of [2] we get that for every $(t, x, y) \in(0, \infty) \times D_{r} \times D_{r}$,

$$
q^{D_{r}}(t, x, y)=p^{D_{r}}(t, x, y)+\int_{0}^{t} \int_{D_{r}} q^{D_{r}}(s, x, z) U(z) \cdot \nabla_{z} p^{D_{r}}(t-s, z, y) d z d s .
$$

Following the same argument in the proof of Theorem 4.2 of [21], using (3.6)-(3.7) and Lemma 3.4 above instead of using (4.1)-(4.3) and Lemma 4.1 of [21], we get that for $(t, x, y) \in(0, \infty) \times D_{r} \times D_{r}$

$$
q^{D_{r}}(t, x, y) \leq M_{8} t^{-\frac{d}{2}} e^{-\frac{M_{4}|x-y|^{2}}{2 t}} \sum_{k=0}^{\infty}\left(M_{8} L_{3}\left(d, M_{4}\right) \sum_{i=1}^{d} N_{U^{i}}^{1}\left(\frac{r^{2}}{M_{4}}\right)\right)^{k}
$$

and

$$
\left|\nabla_{x} q^{D_{r}}(t, x, y)\right| \leq M_{9} t^{-\frac{d+1}{2}} e^{-\frac{M_{4}|x-y|^{2}}{2 t}} \sum_{k=0}^{\infty}\left(M_{9} L_{4}\left(d, M_{4}\right) \sum_{i=1}^{d} N_{U^{i}}^{1}\left(\frac{r^{2}}{M_{4}}\right)\right)^{k} .
$$

Choose $r_{0}$ small such that

$$
M_{8} L_{3}\left(d, M_{4}\right) \sum_{i=1}^{d} N_{U^{i}}^{1}\left(\frac{r_{0}^{2}}{M_{4}}\right)<\frac{1}{2} \quad \text { and } \quad M_{9} L_{4}\left(d, M_{4}\right) \sum_{i=1}^{d} N_{U^{i}}^{1}\left(\frac{r_{0}^{2}}{M_{4}}\right)<\frac{1}{2} .
$$


Then for any $r \leq r_{0}$, we have

$q^{D_{r}}(t, x, y) \leq 2 M_{8} t^{-\frac{d}{2}} e^{-\frac{M_{4}|x-y|^{2}}{2 t}} \quad$ and $\quad\left|\nabla_{x} q^{D_{r}}(t, x, y)\right| \leq 2 M_{9} t^{-\frac{d+1}{2}} e^{-\frac{M_{4}|x-y|^{2}}{2 t}}$.

Using the above estimates we can prove the upper bound on the Green functions when $D$ is shrunk small enough.

Theorem 3.6 Let $D$ be a bounded $C^{1,1}$ domain in $\mathbf{R}^{d}$. Then there exists a constant $r_{1}=r_{1}(D, \mu)>0$ depending on $\mu$ only via the rate at which $\max _{1 \leq i \leq d} M_{\mu^{i}}(r)$ goes to zero such that for $r \leq r_{1}, z \in \mathbf{R}^{d}, x, y \in D_{r}^{z}$,

$$
G_{D_{r}^{z}}(x, y) \leq C_{1}\left(1 \wedge \frac{\rho(x)}{|x-y|}\right)\left(1 \wedge \frac{\rho(y)}{|x-y|}\right) \frac{1}{|x-y|^{d-2}}
$$

where $C_{1}$ only depends on d, diam (D) and the constants in (3.8) and (3.12), and

$$
\left|\nabla_{x} G_{D_{r}^{z}}(x, y)\right| \leq C_{2}\left(1 \wedge \frac{\rho(x)}{|x-y|}\right)\left(1 \wedge \frac{\rho(y)}{|x-y|}\right) \frac{1}{|x-y|^{d-1}}
$$

where $C_{2}$ only depends on $d$, diam (D) and the constants in (3.9) and (3.13).

Proof Recall that $r_{0}$ is the constant $r_{0}$ from Theorem 3.5, and $t_{0}$ is the constant $t_{0}$ used in (3.8) and (3.9). Let $r_{1}:=\sqrt{t}_{0} \wedge r_{0}$ and $l:=\operatorname{diam}(D)$. Note that for every $x, y \in D_{r}^{z},|x-y| \leq r l$. We fix $r \leq r_{1}$ and suppress indices. Using (3.8), we have

$$
\begin{aligned}
& \int_{0}^{r^{2}} q^{D}(t, x, y) d t \leq M_{12} \int_{0}^{r^{2}}\left(1 \wedge \frac{\rho(x)}{\sqrt{t}}\right)\left(1 \wedge \frac{\rho(y)}{\sqrt{t}}\right) t^{-\frac{d}{2}} e^{-\frac{M_{13}|x-y|^{2}}{2 t}} d t \\
& =\frac{M_{12}}{|x-y|^{d-2}} \int_{\frac{|x-y|^{2}}{r^{2}}}^{\infty} u^{\frac{d-4}{2}}\left(1 \wedge \frac{\sqrt{u} \rho(x)}{|x-y|}\right)\left(1 \wedge \frac{\sqrt{u} \rho(y)}{|x-y|}\right) e^{-\frac{1}{2} M_{13} u} d u \\
& \leq \frac{M_{12}}{|x-y|^{d-2}}\left(1 \wedge \frac{\rho(x)}{|x-y|}\right)\left(1 \wedge \frac{\rho(y)}{|x-y|}\right) \int_{0}^{\infty} u^{\frac{d-4}{2}}(u \vee 1) e^{-\frac{1}{2} M_{13} u} d u .
\end{aligned}
$$


On the other hand, by the Chapman-Kolmogorov equation, (3.8) and Theorem 3.5, if $t>r^{2}$ we have

$$
\begin{aligned}
q^{D}(t, x, y)= & \int_{D} \int_{D} q^{D}\left(\frac{r^{2}}{2}, x, z\right) q^{D}\left(t-r^{2}, z, w\right) q^{D}\left(\frac{r^{2}}{2}, w, y\right) d z d w \\
\leq & 2 M_{12}^{2} M_{8} \psi_{D}\left(r^{2}, x, y\right) \int_{D} \int_{D}\left(\frac{r^{2}}{2}\right)^{-d} \\
& \times\left(t-r^{2}\right)^{-\frac{d}{2}} e^{-\frac{M_{13}|x-z|^{2}}{r^{2}}} e^{-\frac{M_{4}|z-w|^{2}}{2\left(t-r^{2}\right)}} e^{-\frac{M_{13}|w-y|^{2}}{r^{2}}} d z d w \\
\leq & 2 M_{12}^{2} M_{8} l^{2} \psi_{D}\left(|x-y|^{2}, x, y\right) \int_{\mathbf{R}^{d} \mathbf{R}^{d}}\left(\frac{r^{2}}{2}\right)^{-d} \\
& \times\left(t-r^{2}\right)^{-\frac{d}{2}} e^{-\frac{M_{13}|x-z|^{2}}{r^{2}}} e^{-\frac{M_{4}|z-w|^{2}}{2\left(t-r^{2}\right)}} e^{-\frac{M_{13}|w-y|^{2}}{r^{2}}} d z d w \\
\leq & C_{0} M_{12}^{2} M_{8} l^{2} \psi_{D}\left(|x-y|^{2}, x, y\right) t^{-\frac{d}{2}} e^{-\frac{C_{1}|x-y|^{2}}{2 t}}
\end{aligned}
$$

where $C_{0}=C_{0}(d)$ and $C_{1}=C_{1}\left(M_{4}, M_{13}\right)$. Since

$$
\int_{r^{2}}^{\infty} t^{-\frac{d}{2}} e^{-\frac{C_{1}|x-y|^{2}}{2 t}} d t=\frac{1}{|x-y|^{d-2}} \int_{0}^{\frac{|x-y|^{2}}{r^{2}}} u^{\frac{d-4}{2}} e^{-\frac{1}{2} C_{1} u} d u
$$

we have

$$
\begin{aligned}
\int_{r^{2}}^{\infty} q^{D}(t, x, y) d t \leq & C_{0} M_{12}^{2} M_{8} l^{2} \frac{1}{|x-y|^{d-2}}\left(1 \wedge \frac{\rho(x)}{|x-y|}\right) \\
& \times\left(1 \wedge \frac{\rho(y)}{|x-y|}\right) \int_{0}^{\infty} u^{\frac{d-4}{2}} e^{-\frac{1}{2} C_{1} u} d u .
\end{aligned}
$$

Combining (3.14) and (3.15), we have proved the upper estimate for the Green functions.

The upper estimate for $\nabla_{x} G_{D_{r}^{z}}(x, y)$ is similar. So we skip the proof here.

Let $G_{D}^{0}$ be the Green function of the Brownian motion $W$ in $D$. By the Green function estimates for Brownian motions, Theorem 3.1 and 3.6 imply that:

Theorem 3.7 Let $D$ be a bounded $C^{1,1}$ domain in $\mathbf{R}^{d}$. Then there exist constants $r_{1}=r_{1}(D, \mu)>0$ and $C=C(D, \mu)>1$ depending on $\mu$ only via the rate at which $\max _{1 \leq i \leq d} M_{\mu^{i}}(r)$ goes to zero such that for $r \leq r_{1}, z \in \mathbf{R}^{d}, x, y \in D_{r}^{z}$,

$$
C^{-1} G_{D_{r}^{z}}^{0}(x, y) \leq G_{D_{r}^{z}}(x, y) \leq C G_{D_{r}^{z}}^{0}(x, y) .
$$


Now we show that the uniform comparability of Green functions (Theorem 3.7) implies the uniform comparability of the corresponding harmonic measures.

Theorem 3.8 Let $D$ be a bounded $C^{1,1}$ domain in $\mathbf{R}^{d}$. Then there exist constants $R=R(D, \mu)>0$ and $C=C(D, \mu)>1$ depending on $\mu$ only via the rate at which $\max _{1 \leq i \leq d} M_{\mu^{i}}(r)$ goes to zero such that for $r \leq R, z \in \mathbf{R}^{d}$ and $x \in D_{r}^{z}$

$$
C^{-1} \mathbf{P}_{x}\left(W_{\tau_{D_{r}^{z}}} \in d y\right) \leq \mathbf{P}_{x}\left(X_{\tau_{D_{r}^{z}}} \in d y\right) \leq C \mathbf{P}_{x}\left(W_{\tau_{D_{r}^{z}}} \in d y\right)
$$

In particular, there exist constants $r_{2}=r_{2}(d, \mu)>0$ and $M_{15}=M_{15}(d, \mu)>1$ depending only on $\mu$ only via the rate at which $\max _{1 \leq i \leq d} M_{\mu^{i}}(r)$ goes to zero such that for $r \leq r_{2}, z \in \mathbf{R}^{d}$ and $x \in B_{r}^{z}:=B(z, r)$

$$
M_{15}^{-1} \mathbf{P}_{x}\left(W_{\tau_{B_{r}^{z}}} \in d y\right) \leq \mathbf{P}_{x}\left(X_{\tau_{B_{r}^{z}}} \in d y\right) \leq M_{15} \mathbf{P}_{x}\left(W_{\tau_{B_{r}^{z}}} \in d y\right)
$$

Proof The idea of the proof is similar to that of Theorem 2.2 in [9]. Fix a bounded $C^{1,1}$ domain $D$ and constants $R:=r_{1}=r_{1}(D, \mu)>0$ and $c=c(D, \mu)>1$ in Theorem 3.7. We also fix $r \leq R$ and $z \in \mathbf{R}^{d}$ and suppress indices on $D_{r}^{z}$. Let $\varphi \geq 0$ is a continuous function on $\partial D$ and let

$$
u(x):=\mathbf{E}_{x}\left[\varphi\left(X_{\tau_{D}}\right)\right],
$$

which is harmonic for $X^{D}$ and continuous on $\bar{D}$ by Propositions 2.3 and 2.8. Choose increasing smooth domains $D_{n}$ with $\overline{D_{n}} \subset D_{n+1}$ and $\cup_{n} D_{n}=D$. Denote $T_{n}:=$ $\inf \left\{t>0: X_{t} \in D_{n}\right\}$. Let $u_{n}(x):=\mathbf{E}_{x}\left[u\left(X_{T_{n}}^{D}\right)\right]$. Then by Proposition 2.10, there exist Radon measures $v_{n}$ supported on $\partial D_{n}$ such that

$$
u_{n}(x)=G_{D} v_{n}(x)=\int_{\partial D_{n}} G_{D}(x, y) v_{n}(d y) .
$$

Recall that $G_{D}^{0}$ is the Green function of the killed Brownian motion in $D$. Let

$$
v_{n}(x):=G_{D}^{0} v_{n}(x)=\int_{\partial D_{n}} G_{D}^{0}(x, y) v_{n}(d y) .
$$

Then by Theorem 3.7,

$$
c^{-1} v_{n}(x) \leq u_{n}(x) \leq c v_{n}(x), \quad x \in D_{n}
$$

Since $D$ is regular for $X$ (see Proposition 2.2), using Proposition 2.3, the remainder of the argument is almost identical to the corresponding argument on the proof of Theorem 2.2 in [9] (with $b_{1} \equiv 0$ there). So we omit it. 
It is well known that the harmonic measure $\mathbf{P}_{x}\left(W_{\tau_{D}} \in d y\right)$ is mutually absolutely continuous with respect to the surface measure on $\partial D$ if $D$ is a bounded $C^{1,1}$ domain. So immediately we have the following from Theorem 3.8 .

Corollary 3.9 The harmonic measure $\mathbf{P}_{x}\left(X_{\tau_{D}} \in d y\right)$ is mutually absolutely continuous with respect to the surface measure on $\partial D$ if $D$ is a bounded $C^{1,1}$ domain.

\section{Boundary Harnack principle in bounded Lipschitz domains}

In this section, we will use the comparability of harmonic measures obtained in the last section to prove a scale invariant version of the boundary Harnack principle for $X$ in bounded Lipschitz domains. Recall that a bounded domain $D$ is said to be Lipschitz if there is a localization radius $r_{0}>0$ and a constant $\Lambda>0$ such that for every $Q \in \partial D$, there is a Lipschitz function $\phi_{Q}: \mathbf{R}^{d-1} \rightarrow \mathbf{R}$ satisfying $\left|\phi_{Q}(x)-\phi_{Q}(z)\right| \leq \Lambda|x-z|$, and an orthonormal coordinate system $C S_{Q}$ with origin at $Q$ such that

$B\left(Q, r_{0}\right) \cap D=B\left(Q, r_{0}\right) \cap\left\{y=\left(y_{1}, \ldots, y_{d-1}, y_{d}\right)=:\left(\tilde{y}, y_{d}\right)\right.$ in $\left.C S_{Q}: y_{d}>\phi_{Q}(\tilde{y})\right\}$

The pair $\left(r_{0}, \Lambda\right)$ is called the characteristics of the Lipschitz domain $D$.

One of the properties of Lipschitz domain is that it satisfies the uniform exterior cone condition. Recall that an open set $D$ in $\mathbf{R}^{d}$ is said to satisfy the uniform exterior cone condition if there exist constants $\eta>0, r$ and a cone

$$
\mathcal{C}:=\left\{x=\left(x_{1}, \ldots, x_{d}\right) \in \mathbf{R}^{d}: x_{d}<0,\left(x_{1}^{2}+\cdots+x_{d-1}^{2}\right)^{\frac{1}{2}}<\eta\left|x_{d}\right|\right\}
$$

such that for every $Q \in \partial D$, there is a cone $\mathcal{C}_{Q}$ with vertex $Q$, isometric to $\mathcal{C}$, such that $\mathcal{C}_{Q} \cap B(Q, r) \subset D^{c}$. If $D$ is a bounded Lipschitz domain with its characteristics $\left(r_{0}, \Lambda\right)$, then it satisfies the uniform exterior cone condition with $\eta=\eta(d, \Lambda), r=r_{0}$. Moreover, by choosing $r_{0}$ smaller if necessary, one can choose cones $\mathcal{C}_{z}:=z+\mathcal{C}$ in $C S_{Q}$ for each $Q \in \partial D$ and $z \in B\left(Q, r_{0}\right) \cap \partial D$ such that $\mathcal{C}_{z} \cap B\left(Q, r_{0}\right) \subset$ $D^{c} \cap B\left(Q, r_{0}\right)$.

In the remainder of this section, we assume $D$ is a bounded Lipschitz domain and fix its characteristics $\left(r_{0}, \Lambda\right)$ and the constant $\eta$. The constant $r_{1}$ will be the constant from Corollary 5.8 in [21] and the constant $r_{2}$ will be the constant from Theorem 3.8. Without loss of generality, we also assume that $\Lambda \geq 1$.

For every $Q \in \partial D$ and $x \in B\left(Q, r_{0}\right) \cap D$, we define

$$
\delta_{Q}(x):=x_{d}-\phi_{Q}(\tilde{x})
$$

where $\left(\tilde{x}, x_{d}\right)$ is the coordinate of $x$ in $C S_{Q}$. Since $D$ is Lipschitz, there exists a constant $c=c(d, \Lambda) \geq 1$ such for every $Q \in \partial D$ and $x \in B\left(Q, r_{0}\right) \cap D$ we have

$$
c^{-1} \delta_{Q}(x) \leq \rho(x) \leq \delta_{Q}(x)
$$

Using (3.16), we can obtain the following result. 
Lemma 4.1 Suppose that $Q \in \partial D$. If $l \geq 1$, then for every $x \in D \cap B\left(Q, r_{0} / 2\right)$ with $4 l \delta_{Q}(x)<r_{0} \wedge r_{2}$,

$$
\mathbf{P}_{x}\left(X_{\tau_{B(x, 2 l \delta} Q^{(x))}} \in D\right) \leq c<1
$$

where $c=c(d, \Lambda)$.

Proof Fix $Q \in \partial D$ and an orthonormal coordinate system $C S_{Q}$ with origin at $Q$ such that

$B\left(Q, r_{0}\right) \cap D=B\left(Q, r_{0}\right) \cap\left\{y=\left(y_{1}, \ldots, y_{d-1}, y_{d}\right)=:\left(\tilde{y}, y_{d}\right)\right.$ in $\left.C S_{Q}: y_{d}>\phi_{Q}(\tilde{y})\right\}$.

For any $x \in B\left(Q, r_{0}\right) \cap D$, we have $x=\left(\tilde{x}, \phi_{Q}(\tilde{x})+\delta_{Q}(x)\right)$ in $C S_{Q}$. Note that for any $x \in D \cap B\left(Q, r_{0}\right)$ with $4 l \delta_{Q}(x)<r_{0} \wedge r_{2}$ we have $B\left(x, 2 l \delta_{Q}(x)\right) \cap D \subset$ $B\left(Q, r_{0}\right) \cap D$. Let $Q_{x}:=\left(\tilde{x}, \phi_{Q}(\tilde{x})\right)$ in $C S_{Q}$. Since $4 l \delta_{Q}(x)<r_{0}$, there exists a cone $\mathcal{C}_{Q_{x}}:=Q_{x}+\mathcal{C}$ with vertex $Q_{x}$ satisfying

$$
\mathcal{C}_{Q_{x}} \cap \partial B\left(x, 2 l \delta_{Q}(x)\right) \subset \mathcal{C}_{Q_{x}} \cap B\left(Q_{x}, r_{0}\right) \subset D^{c} .
$$

So by Theorem 3.8 (note that $4 l \delta_{Q}(x)<r_{2}$ ),

$$
\begin{aligned}
& \mathbf{P}_{x}\left(X_{\left.\tau_{B\left(x, 2 l \delta_{Q^{(x))}}\right.} \notin D\right)} \geq \mathbf{P}_{x}\left(X_{\tau_{B\left(x, 2 l \delta^{(x))}\right.}} \in \partial B\left(x, 2 l \delta_{Q}(x)\right) \cap \mathcal{C}_{Q_{x}}\right)\right. \\
& \\
& \geq M_{15}^{-1} \mathbf{P}_{x}\left(W_{\tau_{B\left(x, 2 Q^{(x))}\right.}} \in \partial B\left(x, 2 \delta_{Q}(x)\right) \cap \mathcal{C}_{Q_{x}}\right) \\
&=M_{15}^{-1} \mathbf{P}_{0}\left(W_{\tau_{B(0,2)}} \in \partial B(0,2) \cap \mathcal{C}_{(0, \ldots, 0,-1)}\right),
\end{aligned}
$$

which is strictly positive since

$$
|\partial B(0,2)| \leq c\left|\partial B(0,2) \cap \mathcal{C}_{(0, \ldots, 0,-1)}\right|
$$

The above lemma and the Harnack principle (Corollary 5.8 of [21]) imply the following Carleson type estimate.

Theorem 4.2 There exists $c_{0}>0$ such that for any $Q \in \partial D$ and any $x=Q+$ $\left(0, \delta_{Q}(x)\right) \in D$ in $C S_{Q}$ with $\delta_{Q}(x)<\frac{1}{10}\left(\frac{r_{0}}{\Lambda} \wedge r_{1} \wedge r_{2}\right)$, and any nonnegative function $u$ which is harmonic with respect to $X$ in $B\left(x, 4 \delta_{Q}(x)\right) \cap D$ and vanishes continuously on $B\left(x, 4 \delta_{Q}(x)\right) \cap \partial D$, we have

$$
u(y) \leq c_{0} u(x), \text { for every } y \in B\left(x, 2 \delta_{Q}(x)\right) \cap D .
$$

Proof The proof of this theorem is similar to that of Theorem III.1.7 in [3]. Fix $Q \in \partial D$ and an orthonormal coordinate system $C S_{Q}$ with origin at $Q$ such that $B\left(Q, r_{0}\right) \cap D=B\left(Q, r_{0}\right) \cap\left\{y=\left(y_{1}, \ldots, y_{d-1}, y_{d}\right)=:\left(\tilde{y}, y_{d}\right)\right.$ in $\left.C S_{Q}: y_{d}>\phi_{Q}(\tilde{y})\right\}$ 
Also we fix $x=Q+\left(0, \delta_{Q}(x)\right) \in D$ in $C S_{Q}$ with $\delta_{Q}(x)<\frac{1}{6}\left(\frac{r_{0}}{\Lambda} \wedge r_{1} \wedge r_{2}\right)$ and a nonnegative function $u$ which is harmonic with respect to $X$ in $B\left(x, 4 \delta_{Q}(x)\right) \cap D$ and vanishes continuously on $B\left(x, 4 \delta_{Q}(x)\right) \cap \partial D$. Note that

$$
B\left(x, 4 \delta_{Q}(x)\right) \cap D \subset B\left(Q, r_{0}\right) \cap D .
$$

For each $y \in B\left(x, 2 \delta_{Q}(x)\right) \cap D$, let $\xi_{y}:=\left(\tilde{y}, \phi_{Q}(\tilde{y})+\delta_{Q}(x)\right)$ in $C S_{Q}$. Since

$$
|\tilde{y}|^{2}+\left|\phi_{Q}(\tilde{y})+\delta_{Q}(x)\right|^{2} \leq 6 \delta_{Q}(x)^{2}+8 \Lambda^{2} \delta_{Q}(x)^{2}<r_{0}^{2},
$$

$\xi_{y}$ is in $B\left(Q, r_{0}\right) \cap D$. Since $\rho(y) \leq|y-x|+\delta_{Q}(x)<3 \delta_{Q}(x)<r_{1}$, using the Harnack principle (Corollary 5.8 of [21]), (4.2) and a standard chain argument, we can show that there exist $\sigma>0$ such that

$$
u(y) \leq c_{1}\left(\frac{\delta_{Q}(y)}{\delta_{Q}(x)}\right)^{-\sigma} u\left(\xi_{y}\right)
$$

(see Exercise 4 in Chap. 3 of [3]). Also, since $\left|x-\xi_{y}\right| \leq|x-y|+\left|y-\xi_{y}\right| \leq 5 \delta_{Q}(x)<$ $r_{1}$ and $\delta_{Q}(x)=\delta_{Q}\left(\xi_{y}\right)$, using the Harnack principle (Corollary 5.8 of [21]) and (4.3), we have

$$
u(y) \leq c_{2}\left(\frac{\delta_{Q}(y)}{\delta_{Q}(x)}\right)^{-\sigma} u(x)
$$

So

$$
\delta_{Q}(y) \leq c_{3}\left(\frac{u(y)}{u(x)}\right)^{-\frac{1}{\sigma}} \delta_{Q}(x) .
$$

We will prove this lemma by contradiction. Lemma 4.1 implies that for every $y \in B\left(x, 2 \delta_{Q}(x)\right) \cap D$, there exists $\varepsilon<1$ such that

$$
u(y) \leq \mathbf{E}_{y}\left[u\left(X_{\tau_{B(y, 2 Q(y))}}\right): X_{\tau_{B(y, 2 \delta} Q^{(y))}} \in D\right] \leq\left(\sup _{\partial B\left(y, 2 \delta_{Q}(y)\right)} u\right) \varepsilon .
$$

Therefore, if there exists $x_{1} \in B\left(x, 2 \delta_{Q}(x)\right) \cap D$ such that $u\left(x_{1}\right) \geq M u(x)$ ( $M$ will be chosen later so that $\left.\left|x_{n}-Q\right|<r_{0} / 2\right)$, there exists $x_{2} \in \partial B\left(x_{1}, 2 \delta_{Q}\left(x_{1}\right)\right)$ such that

$$
u\left(x_{2}\right)=\sup _{\partial B\left(x_{1}, 2 \delta_{Q}\left(x_{1}\right)\right)} u \geq \frac{u\left(x_{1}\right)}{\varepsilon} \geq \frac{M}{\varepsilon} u(x) .
$$

Recursively one can choose a sequence $\left\{x_{n}\right\}_{n \geq 1}$ with $\left|x_{n+1}-x_{n}\right|=2 \delta_{Q}\left(x_{n}\right)$ and

$$
u\left(x_{n}\right) \geq \frac{M}{\varepsilon^{n-1}} u(x),
$$


which implies that $u\left(x_{n}\right) \rightarrow \infty$. But, by (4.4)-(4.5),

$$
\begin{aligned}
& \left|x_{n}-x\right| \leq\left|x_{n}-x_{1}\right|+\left|x_{1}-x\right| \leq \sum_{j=1}^{\infty} 2 \delta_{Q}\left(x_{j}\right)+2 \delta_{Q}(x) \\
& \leq\left(\sum_{j=1}^{\infty} 2 c_{3}\left(\frac{u\left(x_{j}\right)}{u(x)}\right)^{-\frac{1}{\sigma}}+2\right) \delta_{Q}(x) \leq\left(2 c_{3} M^{-\frac{1}{\sigma}} \sum_{j=1}^{\infty} \varepsilon^{\frac{j-1}{\sigma}}+2\right) \delta_{Q}(x),
\end{aligned}
$$

which is less than $4 \delta_{Q}(x)$ if we choose a $M$ with

$$
M>\left(c_{3} \sum_{j=1}^{\infty} \varepsilon^{\frac{j-1}{\sigma}}\right)^{\sigma} .
$$

The above argument also implies that $\delta_{Q}\left(x_{n}\right) \rightarrow 0$. This contradicts the fact that $u$ vanishes continuously on $B\left(x, 4 \delta_{Q}(x)\right) \cap \partial D$.

To prove a scale invariant version of the boundary Harnack principle, we shall follow the "box method" of Bass and Burdzy [3,5]. We adopt the following notation from [3,5]: for every $Q \in \partial D$ with an orthonormal coordinate system $C S_{Q}$ with origin at $Q$ such that $B\left(Q, r_{0}\right) \cap D=B\left(Q, r_{0}\right) \cap\left\{y\right.$ in $\left.C S_{Q}: y_{d}>\phi_{Q}(\tilde{y})\right\}$, we let

$$
\begin{aligned}
\Delta_{Q}(a, R):= & \left\{y \text { in } C S_{Q}: \phi_{Q}(\tilde{y})+a>y_{d}>\phi_{Q}(\tilde{y}),|\tilde{y}|<R\right\}, \\
\partial^{s} \Delta_{Q}(a, R):= & \left\{y \text { in } C S_{Q}: \phi_{Q}(\tilde{y})+\frac{a}{2}>y_{d} \geq \phi_{Q}(\tilde{y}),|\tilde{y}|=R\right\}, \\
\partial^{u} \Delta_{Q}(a, R):=\left\{y \text { in } C S_{Q}: \phi_{Q}(\tilde{y})+a=y_{d},|\tilde{y}|<R\right\} & \cup\left\{y \text { in } C S_{Q}: \phi_{Q}(\tilde{y})+a \geq y_{n} \geq \phi_{Q}(\tilde{y})+\frac{a}{2},|\tilde{y}|=R\right\} .
\end{aligned}
$$

The proofs of the next three lemmas are similar to those of Lemmas 3.1.5-6 and Theorem 3.1.7 in [3] (also see [4,5]). But one has to be more careful since we do not have the scaling property and we use Theorem 3.8 only for small balls. We spell out the details of the proofs of these lemmas for the reader's convenience.

Lemma 4.3 There exist $c_{1}=c_{1}(D)$ and $c_{2}=c_{2}(D)>0$ such that for any $r, R, a<$ $\frac{1}{4}\left(r_{0} \wedge r_{1} \wedge r_{2}\right), Q \in \partial D$, and $y \in \Delta_{Q}(a, r)$, we have

$$
\mathbf{P}_{y}\left(X_{\tau_{Q_{Q}}(a, R)} \in \partial^{s} \Delta_{Q}(a, R)\right) \leq c_{1} e^{-\frac{c_{2}(R-r)}{a}}
$$

Proof We split into two cases. First we assume that $R-r>12 a$. We define $\left\{S_{n}\right\}_{n \geq 1}$, a sequence of stopping times:

$$
S_{1}:=\inf \left\{t>0:\left|X_{t}-X_{0}\right| \geq 2 a\right\} \quad \text { and } \quad S_{n+1}:=S_{n}+S_{1} \circ \theta_{S_{n}} .
$$


Fix $y \in \Delta_{Q}(a, r)$. Since

$$
\mathbf{P}_{y}\left(X_{\tau_{\Delta^{(}(a, R)}} \in \partial^{s} \Delta_{Q}(a, R)\right) \leq \mathbf{P}_{y}\left(\tau_{\Delta_{Q}(a, R)}>S_{\left[\frac{R-r}{4 a}\right]}\right),
$$

it is enough to show that there exists $\epsilon \in(0,1)$ such that for every $n \leq \frac{1}{4 a}(R-r)$,

$$
\mathbf{P}_{y}\left(\tau_{\Delta_{Q}(a, R)}>S_{n+1}\right) \leq \varepsilon \mathbf{P}_{y}\left(\tau_{\Delta_{Q}(a, R)}>S_{n}\right)
$$

In fact, (4.6) implies that there exist $c_{1}=c_{1}(D)$ and $c_{2}=c_{2}(D)>0$ such that

$$
\mathbf{P}_{y}\left(X_{\tau_{Q^{(a, R)}}} \in \partial^{s} \Delta_{Q}(a, R)\right) \leq c \varepsilon^{n} \leq c_{1} e^{-\frac{c_{2}(R-r)}{a}}
$$

Now we prove (4.6). By the strong Markov property,

$$
\begin{aligned}
& \mathbf{P}_{y}\left(\tau_{\Delta_{Q}(a, R)}>S_{n+1}\right) \\
& \quad \leq \mathbf{E}_{y}\left[\mathbf{P}_{X_{S_{n}}}\left(\tau_{\Delta_{Q}(a, R)}>S_{1}\right): \tau_{\Delta_{Q}(a, R)}>S_{n}\right] .
\end{aligned}
$$

If $n \leq \frac{1}{4 a}(R-r)$ and $\tau_{\Delta_{Q}(a, R)}>S_{n}$, then $X_{S_{n}} \in \Delta_{Q}\left(a, \frac{R+r}{2}\right)$. If $z \in \Delta_{Q}\left(a, \frac{R+r}{2}\right)$, with $l:=\frac{a}{\delta_{Q}(z)} \geq 1$ we have $4 l \delta_{Q}(z)=4 a<\left(r_{0} \wedge r_{1} \wedge r_{2}\right)$. So by Lemma 4.1, we have

$$
\mathbf{P}_{z}\left(\tau_{\Delta_{Q}(a, R)}>S_{1}\right) \leq \mathbf{P}_{z}\left(X_{S_{1}} \in D\right) \leq \varepsilon<1
$$

Therefore (4.6) is true.

If $R-r \leq 12 a$, choose $c_{1}$ larger if necessary so that $c_{1} e^{-\frac{c_{2}(R-r)}{a}} \geq 1$.

Lemma 4.4 There exist $c=c(D)>0$ and $\sigma=\sigma(D)>0$ such that for every $r<\frac{1}{4}\left(r_{0} \wedge r_{1} \wedge r_{2}\right), a<2 r, Q \in \partial D$, and $y \in \Delta_{Q}(r, a)$, we have

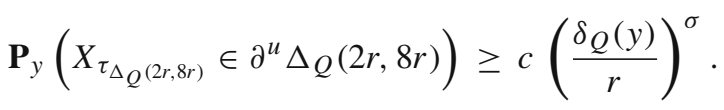

Proof Fix $Q \in \partial D, r<\frac{1}{4}\left(r_{0} \wedge r_{1} \wedge r_{2}\right), a<2 r$ and $y \in \Delta_{Q}(r, a)$. (4.2) says that there exists a constant $c_{1}=c_{1}(D) \geq 1$ such that $c_{1}^{-1} \delta_{Q}(x) \leq \rho(x) \leq \delta_{Q}(x)$ for all $x \in D \cap B\left(Q, r_{0}\right)$. Let $y_{0}=y$ and choose $y_{i}$ above $y_{0}$ in $C S_{Q}$ such that $\left|y_{i}-y_{i-1}\right|=\rho\left(y_{i-1}\right) / 4$. Let $n$ and $m$ be the smallest integers such that $\delta_{Q}\left(y_{n}\right)>2 r$ and $m \geq 8 c_{1}$, respectively. We see that

$$
\delta_{Q}\left(y_{m}\right)=\delta_{Q}\left(y_{0}\right)+\left|y_{0}-y_{1}\right|+\cdots+\left|y_{m-1}-y_{m}\right| \geq 2 \delta_{Q}\left(y_{0}\right) .
$$


By induction, we get $\delta_{Q}\left(y_{k m}\right) \geq 2^{k} \delta_{Q}\left(y_{0}\right)$. Let $k$ be the smallest integer such $2^{k} \delta_{Q}\left(y_{0}\right)>2 r$. Then by the choices of $n, m$ and $k$, we get

$$
n \leq m k \leq\left(8 c_{1}+1\right) \log _{2}\left(\frac{4 r}{\delta_{Q}\left(y_{0}\right)}\right) \leq-\frac{9 c_{1}}{\ln 2} \ln \frac{\delta_{Q}\left(y_{0}\right)}{4 r} .
$$

On the other hand,

$$
\delta_{Q}\left(y_{n}\right)=\delta_{Q}\left(y_{n-1}\right)+\left|y_{n-1}-y_{n}\right| \leq 2 r+\frac{\rho\left(y_{n-1}\right)}{4} \leq 2 r+\frac{\delta_{Q}\left(y_{n-1}\right)}{4} \leq \frac{5 r}{2} .
$$

So $2 r \leq \delta_{Q}\left(y_{n}\right) \leq \frac{5 r}{2}$. Let

$$
A_{j}:=\bigcup_{i=0}^{j} B\left(y_{i}, \frac{\rho\left(y_{i}\right)}{4}\right)
$$

Since $a+\delta_{Q}\left(y_{n}\right)<5 r$.

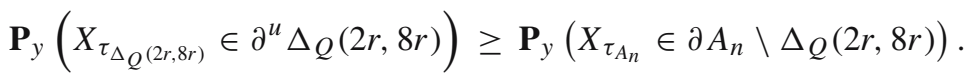

By Theorem 3.8, we also have

$$
\begin{aligned}
& \mathbf{P}_{y_{n}}\left(X_{\tau_{A_{n}}} \in \partial A_{n} \backslash \Delta_{Q}(2 r, 8 r)\right) \\
& \quad \geq \mathbf{P}_{y_{n}}\left(X_{\left.\tau_{B\left(y_{n}, \frac{1}{4} \rho\left(y_{n}\right)\right)} \in \partial B\left(y_{n}, \frac{1}{4} \rho\left(y_{n}\right)\right) \backslash\left(A_{n-1} \cup \Delta_{Q}(2 r, 8 r)\right)\right)}\right. \\
& \geq M_{15}^{-1} \mathbf{P}_{y_{n}}\left(W_{\tau_{B\left(y_{n}, \frac{1}{4} \rho\left(y_{n}\right)\right)}} \in \partial B\left(y_{n}, \frac{1}{4} \rho\left(y_{n}\right)\right) \backslash\left(A_{n-1} \cup \Delta_{Q}(2 r, 8 r)\right)\right) \geq c_{2}>0 .
\end{aligned}
$$

Now, since $\delta_{Q}\left(y_{n}\right)<\frac{5}{2} r<r_{1}$, using the Harnack principle (Corollary 5.8 of [21]) and a standard chain argument we can show that

$$
\mathbf{P}_{y}\left(X_{\tau_{A_{n}}} \in \partial A \backslash \Delta_{Q}(2 r, 8 r)\right) \geq c_{2} c_{3}^{n} .
$$

Therefore, with (4.7) we conclude that

$$
\mathbf{P}_{y}\left(X_{\tau_{\Delta_{Q}(2 r, 8 r)}} \in \partial^{u} \Delta_{Q}(2 r, 8 r)\right) \geq c_{2} c_{3}^{n} \geq c\left(\frac{\delta_{Q}(y)}{r}\right)^{\sigma} .
$$

Lemma 4.5 There exists $c=c(D)>0$ such that for every $r<\frac{1}{4}\left(r_{0} \wedge r_{1} \wedge r_{2}\right)$, $Q \in \partial D$ and $y \in \Delta_{Q}(r, r)$, we have

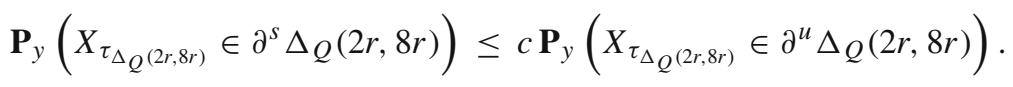


Proof We fix $r<\frac{1}{4}\left(r_{0} \wedge r_{1} \wedge r_{2}\right)$ and $Q \in \partial D$. Let

$$
\Omega_{1}:=\left\{X_{\tau_{\Delta_{Q}(2 r, 8 r)}} \in \partial^{s} \Delta_{Q}(2 r, 8 r)\right\}, \quad \Omega_{2}:=\left\{X_{\tau_{\Delta_{Q}(2 r, 8 r)}} \in \partial^{u} \Delta_{Q}(2 r, 8 r)\right\}
$$

and $\Delta:=\Delta_{Q}(2 r, 8 r)$. We define a decreasing sequence $\left\{r_{i}\right\}_{i \geq 1}$ by

$$
r_{i}:=2 r\left(\frac{3}{4}-\frac{1}{40} \sum_{j=1}^{i} \frac{1}{j^{2}}\right)>r .
$$

Note that $r<\cdots<r_{i+1}<r_{i}<\cdots<r_{1}<\frac{3 r}{2}$. We also define

$$
A_{i}:=\Delta_{Q}\left(2^{-i+1} r, r_{i}\right) \backslash \Delta_{Q}\left(2^{-i} r, r_{i}\right) \quad \text { and } \quad l_{i}:=\sup _{y \in A_{i}} \frac{\mathbf{P}_{y}\left(\Omega_{1}\right)}{\mathbf{P}_{y}\left(\Omega_{2}\right)} .
$$

We claim that $\sup _{i \geq 1} l_{i}<\infty$. First, by Lemma 4.4 , we get $l_{1}<\infty$. Let $\tau_{i}:=$ $\tau_{\Delta_{Q}\left(2^{-i+1} r, r_{i-1}\right)}$ and $y \in A_{i}$. We then have:

$$
\begin{aligned}
\mathbf{P}_{y}\left(\Omega_{1}\right) \leq & \mathbf{P}_{y}\left(X_{\tau_{i}} \in \partial^{s} \Delta_{Q}\left(2^{-i+1} r, r_{i-1}\right)\right) \\
& +\mathbf{P}_{y}\left(X_{\tau_{i}} \in \partial^{u} \Delta_{Q}\left(2^{-i+1} r, r_{i-1}\right) ; \Omega_{1}\right):=J_{i, 1}+J_{i, 2} .
\end{aligned}
$$

Since $r_{i-1}-r_{i} \leq \frac{r}{20 i^{2}}$, by Lemma $4.3, J_{i, 1}$ is bounded above by

$$
c_{1} e^{-c_{2} \frac{\left.r(20)^{2}\right)}{2^{-i} i_{r}}} \leq c_{1} e^{-c_{3} \frac{2^{i}}{i^{2}}} \leq c_{4} \frac{2^{-i \sigma}}{i^{2}} \leq \frac{c_{5}}{i^{2}}\left(\frac{\delta_{Q}(y)}{r}\right)^{\sigma}
$$

which is, in turn, bound above by $\frac{c}{i^{2}} \mathbf{P}_{y}\left(\Omega_{2}\right)$ by Lemma 4.4. On the other hand, using the strong Markov property (twice), $J_{i, 2}$ is bounded above by

$$
\begin{aligned}
& \mathbf{E}_{y}\left[\mathbf{P}_{X_{\tau_{i}}}\left(\Omega_{1}\right): \partial^{u} \Delta_{Q}\left(2^{-i+1} r, r_{i-1}\right)\right] \\
& \quad \leq l_{i-1} \mathbf{P}_{y}\left(X_{\tau_{i}} \in \partial^{u} \Delta_{Q}\left(2^{-i+1} r, r_{i-1}\right) ; \Omega_{2}\right) \leq l_{i-1} \mathbf{P}_{y}\left(\Omega_{2}\right) .
\end{aligned}
$$

Therefore

$$
\mathbf{P}_{y}\left(\Omega_{1}\right) \leq \frac{c}{i^{2}} \mathbf{P}_{y}\left(\Omega_{2}\right)+l_{i-1} \mathbf{P}_{y}\left(\Omega_{2}\right) .
$$

Divide the above by $\mathbf{P}_{y}\left(\Omega_{2}\right)$ and take the supremum over $y \in A_{i}$. We get

$$
l_{i} \leq \frac{c}{i^{2}}+l_{i-1},
$$

which implies that $\sup _{i \geq 1} l_{i}<\infty$.

The following is the main result of this section. 
Theorem 4.6 (Scale invariant boundary Harnack principle) There exist constants $M, c>1$ and $r_{3}>0$, depending on $\mu$ only via the rate at which $\max _{1 \leq i \leq d} M_{\mu^{i}}(r)$ goes to zero such that for every $Q \in \partial D, r<r_{3}$ and any nonnegative functions $u$ and $v$ which are harmonic with respect to $X$ in $D \cap B(Q, M r)$ and vanish continuously on $\partial D \cap B(Q, M r)$, we have

$$
\frac{u(x)}{v(x)} \leq c \frac{u(y)}{v(y)} \quad \text { for any } x, y \in D \cap B\left(Q, \frac{r}{1+\Lambda}\right)
$$

Proof Let $A:=10 \sqrt{1+\Lambda^{2}}, M:=10 A$ and $r_{3}:=\frac{1}{M}\left(r_{0} \wedge r_{1} \wedge r_{2}\right)$. Fix $r<r_{3}$ and $Q \in \partial D$ with an orthonormal coordinate system $C S_{Q}$ with origin at $Q$ such that $B\left(Q, r_{0}\right) \cap D=B\left(Q, r_{0}\right) \cap\left\{y\right.$ in $\left.C S_{Q}: y_{d}>\phi_{Q}(\tilde{y})\right\}$. Note that if $y \in B\left(Q, \frac{r}{1+\Lambda}\right) \cap$ $D$, then

$$
y_{d}-\phi_{Q}(\tilde{y}) \leq y_{d}+\Lambda|\tilde{y}|<\frac{r}{1+\Lambda}+\Lambda \frac{r}{1+\Lambda}=r .
$$

So $B\left(Q, \frac{r}{1+\Lambda}\right) \cap D \subset \Delta_{Q}(r, r)$. Let $\Delta:=\Delta_{Q}(2 r, 8 r), \partial^{s} \Delta:=\partial^{s} \Delta_{Q}(2 r, 8 r)$ and $\partial^{u} \Delta:=\partial^{u} \Delta_{Q}(2 r, 8 r)$. Assume $x, y \in \Delta_{Q}(r, r)$ and let $a=Q+(\tilde{0}, A r)$. By the Harnack principle (Corollary 5.8 of [21]),

$$
\sup _{\partial^{u} \Delta} u \leq c_{1} u(a) \text { and } \inf _{\partial^{u} \Delta} v \geq c_{2} v(a) .
$$

If $z \in \bar{\Delta}$,

$$
\begin{aligned}
|a-z| & \leq|a-Q|+|Q-z| \leq A r+\left|\left(\tilde{z}, \phi_{Q}(\tilde{z})\right)-(\tilde{Q}, 0)\right|+2 r \\
& \leq A r+8 r \sqrt{1+\Lambda^{2}}+2 r<2 A r .
\end{aligned}
$$

So $\bar{\Delta} \subset B(a, 2 A r)$. Clearly $B(a, 4 A r) \subset B(Q, M r)$. Thus by Theorem 4.2,

$$
\sup _{\partial^{s} \Delta} u \leq c_{3} u(a) .
$$

Therefore by (4.9), (4.10) and Lemma 4.5, for every $x \in \Delta_{Q}(r, r)$,

$$
\begin{aligned}
u(x) & =\mathbf{E}_{x}\left[u\left(X_{\tau_{\Delta}}\right): X_{\tau_{\Delta}} \in \partial^{s} \Delta\right]+\mathbf{E}_{x}\left[u\left(X_{\tau_{\Delta}}\right): X_{\tau_{\Delta}} \in \partial^{u} \Delta\right] \\
& \leq c_{3} u(a) \mathbf{P}_{x}\left(X_{\tau_{\Delta}} \in \partial^{s} \Delta\right)+c_{1} u(a) \mathbf{P}_{x}\left(X_{\tau_{\Delta}} \in \partial^{u} \Delta\right) \\
& \leq c_{4} \frac{u(a)}{v(a)} v(a) \mathbf{P}_{x}\left(X_{\tau_{\Delta}} \in \partial^{u} \Delta\right) \\
& \leq c_{5} \frac{u(a)}{v(a)} \mathbf{E}_{x}\left[v\left(X_{\tau_{\Delta}}\right): X_{\tau_{\Delta}} \in \partial^{u} \Delta\right] \leq c_{5} \frac{u(a)}{v(a)} v(x) .
\end{aligned}
$$


The above argument also implies that

$$
\frac{v(y)}{u(y)} \leq c_{5} \frac{v(a)}{u(a)}
$$

Therefore

$$
\frac{u(x)}{v(x)} \leq c_{5} \frac{u(a)}{v(a)} \leq c_{5}^{2} \frac{u(y)}{v(y)}
$$

\section{Martin kernel and Martin boundary}

By using Green function estimates, we proved in [21] that, for any bounded $C^{1,1}$ domain $D$, both the Martin boundary and the minimal Martin boundary with respect to $X$ in $D$ coincide with the Euclidean boundary. In this section, we will extend this result to bounded Lipschitz domains by using the results of the previous sections.

Fix $x_{0} \in D$ throughout this section and define

$$
M_{D}(x, y):= \begin{cases}\frac{G_{D}(x, y)}{G_{D}\left(x_{0}, y\right)}, & \text { if } x \in D \text { and } y \in D \backslash\left\{x_{0}\right\} \\ 1_{\left\{x_{0}\right\}}(x), & \text { if } y=x_{0} .\end{cases}
$$

By Theorem 2.9 (1), we know that for each $y \in D \backslash\left\{x_{0}\right\}$ and $\varepsilon>0, M_{D}(\cdot, y)$ is a harmonic function with respect to $X$ in $D \backslash B(y, \varepsilon)$ and

$$
M_{D}(x, y)=\mathbf{E}_{x}\left[M_{D}\left(X_{\tau_{D \backslash B(y, \varepsilon)}^{D}}^{D}, y\right)\right], \quad x \in D \backslash B(y, \varepsilon) .
$$

Using the Riesz decomposition theorem (Theorem 2.9 (2)-(3)), Proposition 2.10, the Harnack inequality (Corollary 5.8 in [21]) and the Hölder continuity of harmonic functions (Theorem 5.5 in [21]), one can follow the arguments in [22] (see also Sect. 2.7 of [3] or [25]) to show that the process $X^{D}$ has a Martin boundary $\partial_{M} D$ satisfying the following properties.

(M1) $\quad D \cup \partial_{M} D$ is a compact metric space;

(M2) $D$ is open and dense in $D \cup \partial_{M} D$ and its relative topology coincides with its original topology;

(M3) $M_{D}(x, \cdot)$ can be extended to $\partial_{M}$ uniquely in such a way that, $M_{D}(x, y)$ converges to $M_{D}(x, w)$ as $y \rightarrow w \in \partial_{M} D$, the function $M_{D}(x, w)$ is jointly continuous on $D \times \partial_{M} D$, and $M_{D}\left(\cdot, w_{1}\right) \neq M_{D}\left(\cdot, w_{2}\right)$ if $w_{1} \neq w_{2}$;

The Harnack inequality (Corollary 5.8 in [21]) and the harmonicity of $M_{D}(\cdot, y)$ in $D \backslash\{y\}$ imply the harmonicity of $M_{D}(\cdot, w)$ in $D$ for $w \in \partial_{M} D$.

Proposition 5.1 For every $w \in \partial_{M} D, x \mapsto M(x, w)$ is harmonic with respect to $X$ in $D$. 
Proof Fix $w \in \partial_{M} D$ and relatively compact open sets $U \subset \bar{U} \subset U_{1} \subset \overline{U_{1}}$ in $D$. Let $\delta:=\frac{1}{2} \operatorname{dist}\left(U, \partial U_{1}\right)$. Choose a sequence $\left\{y_{n}\right\}_{n \geq 1}$ in $D \backslash \overline{U_{1}}$ converging to $w$ in $D \cup \partial_{M} D$ so that

$$
M_{D}(x, w)=\lim _{n \rightarrow \infty} M_{D}\left(x, y_{n}\right)
$$

Since $M_{D}\left(\cdot, y_{n}\right)$ is harmonic in a neighborhood of $U$ for every $n \geq 1$, we have

$$
\mathbf{E}_{x}\left[M_{D}\left(X_{\tau_{U}}^{D}, y_{n}\right)\right]=M_{D}\left(x, y_{n}\right), \quad x \in U
$$

Using the Harnack inequality (Corollary 5.8 in [21]) and a Harnack chain argument, we have for every $z \in \partial U$,

$$
M_{D}\left(z, y_{n}\right)=\frac{G_{D}\left(z, y_{n}\right)}{G_{D}\left(x_{0}, y_{n}\right)} \leq c_{1} \frac{G_{D}\left(x_{0}, y_{n}\right)}{G_{D}\left(x_{0}, y_{n}\right)}=c_{1}, \quad n \geq 1,
$$

for some $c_{1}=c_{1}(\delta, D)>0$. Thus by the bounded convergence theorem,

$$
\lim _{n \rightarrow \infty} \mathbf{E}_{x}\left[M_{D}\left(X_{\tau_{U}}^{D}, y_{n}\right)\right]=\mathbf{E}_{x}\left[M_{D}\left(X_{\tau_{U}}^{D}, w\right)\right]=M_{D}(x, w), \quad x \in U .
$$

Since $M_{D}\left(x_{0}, y\right)=1$ for every $y \in D \cup \partial_{M} D \backslash\left\{x_{0}\right\}$, using Theorem 5.5 and Corollary 5.8 in [21] we can show that, for any compact subset $K$ of $D$, the family $\left\{M_{D}(\cdot, w): w \in \partial_{M} D\right\}$ is uniformly bounded and equicontinuous on $K$. One can then apply the Ascoli-Arzelà theorem to prove the existence of minimal Martin boundary $\partial_{m} D$. We omit the details since the proof will be almost identical to the classical case (see Sect. 2.7 of [3]). Thus, by combining the above argument with Theorem 2.9, we have that for every excessive function $f$ of $X^{D}$, there are a unique Radon measure $v_{1}$ on $D$ and a unique finite measure $\nu_{2}$ on $\partial_{m} D$ such that

$$
f(x)=\int_{D} G_{D}(x, y) v_{1}(d y)+\int_{\partial_{m} D} M_{D}(x, z) v_{2}(d z),
$$

and $f$ is harmonic in $D$ with respect to $X$ if and only if $v_{1}=0$. When $f$ is harmonic in $D$ with respect to $X$, the measure $v_{2}$ above is called the Martin measure of $f$.

From now on we shall assume that $D$ is a bounded Lipschitz domain. First, we will use Carleson estimate (Theorem 4.2) to show that there exists a continuous map from the Martin compactification $\partial_{M} D \cup D$ onto the Euclidean closure $\bar{D}$.

Lemma 5.2 There exists a continuous map ` from $\partial_{M} D \cup D$ onto $\bar{D}$ which is an identity map in $D$.

Proof Note that $D \cup \partial_{M} D$ is a compact metric space. We will show that if a sequence $\left\{y_{n}\right\}_{n \geq 1}$ in $D$ converges to a point $w$ in $\partial_{M} D$, it converges in $\bar{D}$. Assume that a subsequence $\left\{y_{n_{k}}\right\}_{k \geq 1}$ of $\left\{y_{n}\right\}_{n \geq 1}$ converges to $y_{0} \in \bar{D}$. Let $U$ and $U_{0}$ be relatively compact 
open sets with $y_{0} \in U_{0} \subset \overline{U_{0}} \subset U \subset \bar{U} \subset D \backslash\left\{x_{0}\right\}$. Choose $k_{0}$ large so that $y_{n_{k}} \in U_{0}$ for every $k \geq k_{0}$ and let $\delta:=\operatorname{dist}\left(U_{0}, \partial U\right)$. Suppose $x \in D \backslash \bar{U}$. By the harmonicity of $G_{D}\left(\cdot, y_{n_{k}}\right)$ on $D \backslash U_{0}$ and Theorem 2.6, we can use the Carleson estimate (Theorem 4.2) and a Harnack chain argument (Corollary 5.8 in [21]) with $G_{D}\left(\cdot, y_{n_{k}}\right.$ ) on $D \backslash U_{0}$. Thus for every $a \in D \cap \partial U$,

$$
M_{D}\left(a, y_{n_{k}}\right)=\frac{G_{D}\left(a, y_{n_{k}}\right)}{G_{D}\left(x_{0}, y_{n_{k}}\right)} \leq c_{1} \frac{G_{D}\left(x_{0}, y_{n_{k}}\right)}{G_{D}\left(x_{0}, y_{n_{k}}\right)}=c_{1}, \quad k \geq k_{0} \text {, }
$$

for some $c_{1}=c_{1}(\delta, D)$. Therefore, using (5.1) and the bounded convergence theorem, we have for every $x \in D \backslash U$

$$
\begin{aligned}
M_{D}(x, w) & =\lim _{k \rightarrow \infty} M_{D}\left(x, y_{n_{k}}\right)=\lim _{k \rightarrow \infty} \mathbf{E}_{x}\left[M_{D}\left(X_{\tau_{D \backslash U}}^{D}, y_{n_{k}}\right)\right] \\
& =\mathbf{E}_{x}\left[M_{D}\left(X_{\tau_{D \backslash U}}^{D}, w\right)\right] .
\end{aligned}
$$

(5.3) implies that $M_{D}(a, w) \leq c_{1}$ for $a \in D \cap \partial U$. So we have for every $y \in \partial D \backslash \bar{U}$,

$$
\lim _{x \rightarrow y} M_{D}(x, w) \leq c_{1} \lim _{x \rightarrow y} \mathbf{P}_{x}\left(\tau_{D \backslash U}<\tau_{D}\right)=0, \quad y \in \partial D \backslash \bar{U}
$$

If there exists a subsequence of $\left\{y_{n}\right\}_{n \geq 1}$ converging to a point in $\bar{D}$ different from $y_{0}$, the above argument says that the Martin kernel would vanish continuously on $\partial D$. But that implies that $M_{D}(\cdot, w) \equiv 0$ by the maximum principle (Lemma 7.2 in [21]), which is impossible. Therefore $y_{0} \in \bar{D}$ must be unique. Moreover, $y_{0}$ must be in $\partial D$ otherwise we could choose $U$ in $D$ and also argue that the Martin kernel would vanish continuously on $\partial D$.

Since $\left\{y_{n}\right\}_{n \geq 1}$ is bounded in $\bar{D}$, the above argument shows that every subsequence of $\left\{y_{n}\right\}_{n \geq 1}$ has a further subsequence converging to a unique point in $\bar{D}$. So the map $\iota$ defined by $\iota(w)=y_{0}$ is continuous.

Now we show that $\iota$ is onto. Fix a point $z_{0} \in \partial D$ and choose a sequence $\left\{y_{n}\right\}_{n \geq 1}$ in $D$ converging to a $z_{0}$ in $\bar{D}$. Since $\left\{y_{n}\right\}_{n \geq 1}$ is a sequence in the compact metric space $D \cup \partial_{M} D$, there exists a subsequence $\left\{y_{n_{k}}\right\}_{k \geq 1}$ of $\left\{y_{n}\right\}_{n \geq 1}$ converges to a $w_{0} \in D \cup \partial_{M} D$. By the continuity of $\iota, \iota\left(w_{0}\right)=z_{0}$.

From the proof of the above lemma, we have the following corollary.

Corollary 5.3 Suppose $w_{0} \in \partial_{M} D$. For every open subset $V \ni \iota\left(w_{0}\right)$, we have,

$$
M_{D}\left(x, w_{0}\right)=\mathbf{E}_{x}\left[M_{D}\left(X_{\tau_{D \backslash V}}^{D}, w_{0}\right)\right]
$$

If $z \in \partial D$ is different from $\iota\left(w_{0}\right)$, then

$$
\lim _{D \ni x \rightarrow z} M_{D}\left(x, w_{0}\right)=0 .
$$


Lemma 5.4 For each $w_{0} \in \partial_{M} D$, the support of the Martin measure $v$ for $M_{D}\left(\cdot, w_{0}\right)$ is contained in $\iota^{-1}\left(\iota\left(w_{0}\right)\right)$.

Proof Let $z_{0}:=\iota\left(w_{0}\right)$ and $v$ be the Martin measure $v$ for $M_{D}\left(\cdot, w_{0}\right)$. For any closed subset $U$ of $\partial_{M} D$ such that $U \cap \iota^{-1}\left(z_{0}\right)=\emptyset$, define

$$
h(x)=\int_{U} M_{D}(x, w) v(d w) \quad \text { for } x \in D .
$$

We will show that $h \equiv 0$, which implies that the support of $v$ is contained in $\iota^{-1}\left(z_{0}\right)$.

If $z \in \partial D$ is different from $z_{0}=\iota\left(w_{0}\right)$, then by Corollary 5.3,

$$
h(x) \leq \int_{\partial_{M} D} M_{D}(x, w) v(d w)=M_{D}\left(x, w_{0}\right) \rightarrow 0 \quad \text { as } x \rightarrow z
$$

On the other hand, for any $w \in U$, by Corollary $5.3, \lim _{x \rightarrow z_{0}} M_{D}(x, w)=0$. Let $\delta:=\operatorname{dist}\left(z_{0}, \iota(U)\right)$. By the Carleson estimate (Theorem 4.2) and a Harnack chain argument (Corollary 5.8 in [21]) we get that for every $x \in B\left(z_{0}, \frac{1}{3} \delta\right)$ and $y \in\{z \in D$ : $\left.\operatorname{dist}(z, \iota(U))<\frac{1}{3} \delta\right\}$,

$$
\frac{G_{D}(x, y)}{G_{D}\left(x_{0}, y\right)} \leq c_{1} \frac{G_{D}\left(x_{0}, y\right)}{G_{D}\left(x_{0}, y\right)}=c_{1}
$$

for some $c_{1}=c_{1}(\delta, D)$. Thus, for any $w \in U$ and $x \in B\left(z_{0}, \frac{1}{3} \delta\right), M_{D}(x, w)$ is bounded above by $c_{1}$. Therefore by the bounded convergence theorem, we get

$$
\lim _{x \rightarrow z_{0}} h(x)=\lim _{x \rightarrow z_{0}} \int_{U} M_{D}(x, w) v(d w)=0 .
$$

Combining (5.4), (5.5) and Maximum principle, we conclude that $h \equiv 0$

The next lemma is a consequence of the scale invariant version of the boundary Harnack principle (Theorem 4.6).

Lemma 5.5 There exists constant $c_{1}>1$ such that for every $Q \in \partial D$ and any nonnegative functions $u$ and $v$ which are harmonic with respect to $X$ in $D$ and vanish continuously on $\partial D \backslash\{Q\}$, we have

$$
\frac{u(x)}{u\left(x_{0}\right)} \leq c_{1} \frac{v(x)}{v\left(x_{0}\right)} \quad \text { for any } x \in D
$$

Proof Recall the constants $M, c>1$ and $r_{3}>0$ in Theorem 4.6 and the Lipschitz constant $\Lambda$. We fix $M, c>1, r_{3}>0, \Lambda$ and a point $Q$ on $\partial D$ throughout this 
proof. We also fix an orthonormal coordinate system $C S_{Q}$ with origin at $Q$ such that $B\left(Q, r_{0}\right) \cap D=B\left(Q, r_{0}\right) \cap\left\{y\right.$ in $\left.C S_{Q}: y_{d}>\phi(\tilde{y})\right\}$. Let $r<\frac{r_{3}}{4}, Q_{r}:=\left(\tilde{0}, \frac{r}{2(1+\Lambda)}\right)$,

$$
\begin{aligned}
\Delta_{r} & :=\left\{y \text { in } C S_{Q}: \phi(\tilde{y})+\frac{r}{2(1+\Lambda)}>y_{d}>\phi(\tilde{y}),|\tilde{y}|<2 M r\right\}, \\
\partial^{s} \Delta_{r} & :=\left\{y \text { in } C S_{Q}: \phi(\tilde{y})+\frac{r}{2(1+\Lambda)} \geq y_{d}>\phi(\tilde{y}),|\tilde{y}|=2 M r\right\}, \\
\partial^{u} \Delta_{r} & :=\left\{y \text { in } C S_{Q}: \phi(\tilde{y})+\frac{r}{2(1+\Lambda)}=y_{d},|\tilde{y}| \leq 2 M r\right\} .
\end{aligned}
$$

Choose $r$ smaller if necessary so that $x_{0} \notin \overline{\Delta_{r}}$. For $|\tilde{y}|=2 M r$, we have $|(\tilde{y}, \phi(\tilde{y}))|>$ $M r$. So $u$ and $v$ are harmonic with respect to $X$ in $D \cap B((\tilde{y}, \phi(\tilde{y})), M r)$ and vanish continuously on $\partial D \cap B((\tilde{y}, \phi(\tilde{y})), M r)$ where $|\tilde{y}|=2 M r$. Therefore by Theorem 4.6,

$$
\frac{u(x)}{v(x)} \leq c \frac{u(y)}{v(y)} \quad \text { for any } x, y \in \partial^{s} \Delta_{r} \text { with } \tilde{x}=\tilde{y} .
$$

We claim that there exists $c_{3}$ such that for every $x \in \partial^{s} \Delta_{r} \cup \partial^{u} \Delta_{r}$

$$
c_{3}^{-1} \frac{u(x)}{u\left(Q_{r}\right)} \leq \frac{v(x)}{v\left(Q_{r}\right)} \leq c_{3} \frac{u(x)}{u\left(Q_{r}\right)} .
$$

If $x \in \partial^{u} \Delta_{r}$, the Harnack principle (Corollary 5.8 in [21]) implies that there exists constant $c_{2}>1$ such that

$$
c_{2}^{-1}<\frac{u(x)}{u\left(Q_{r}\right)}, \frac{v(x)}{v\left(Q_{r}\right)}<c_{2} .
$$

Thus (5.8) is true for every $x \in \partial^{u} \Delta_{r}$. Now we assume that $x \in \partial^{s} \Delta_{r}$. Let $x_{r}:=$ $\left(\tilde{x}, \phi(\tilde{x})+\frac{r}{2(1+\Lambda)}\right)$. Since $x_{r} \in \partial^{s} \Delta_{r},(5.7)$ implies that

$$
c^{-1} \frac{u(x)}{u\left(x_{r}\right)} \leq \frac{v(x)}{v\left(x_{r}\right)} \leq c \frac{u(x)}{u\left(x_{r}\right)} .
$$

On the other hand, $x_{r}$ is also in $\partial^{u} \Delta_{r}$. Thus, by (5.9),

$$
c_{2}^{-1}<\frac{u\left(x_{r}\right)}{u\left(Q_{r}\right)}, \frac{v\left(x_{r}\right)}{v\left(Q_{r}\right)}<c_{2}
$$

(5.10)-(5.11) imply that (5.8) is true for each $x \in \partial^{s} \Delta_{r}$ and $c_{3}$ is independent of the choice of $x \in \partial^{s} \Delta_{r}$. Therefore (5.8) is true for every $x \in \partial^{s} \Delta_{r} \cup \partial^{u} \Delta_{r}$. Moreover, by applying the maximum principle (Lemma 7.2 in [21]), (5.8) is true for every $x \in D \backslash \Delta_{r}$. In particular, $\frac{u\left(x_{0}\right)}{u\left(Q_{r}\right)} \geq c_{3}^{-1} \frac{v\left(x_{0}\right)}{v\left(Q_{r}\right)}$. Therefore

$$
\frac{u(x)}{u\left(x_{0}\right)}=\frac{u(x)}{u\left(Q_{r}\right)} \frac{u\left(Q_{r}\right)}{u\left(x_{0}\right)} \leq c_{3}^{2} \frac{v(x)}{v\left(Q_{r}\right)} \frac{v\left(Q_{r}\right)}{v\left(x_{0}\right)}=c_{3}^{2} \frac{v(x)}{v\left(x_{0}\right)}, \quad x \in D \backslash \Delta_{r} .
$$


Since $c_{3}$ is independent of $r$, by letting $r \downarrow 0$, the above inequality is true for every $x \in D$.

Lemma 5.6 เ is one to one. Moreover $\partial_{m} D=\partial_{M} D$.

Proof Fix $z_{0} \in \partial D$. (5.2) and Lemma 5.4 imply that for each $w \in \iota^{-1}\left\{z_{0}\right\}$, there is a unique Martin measure $v_{w}$ for $M_{D}(\cdot, w)$ such that

$$
M_{D}(x, w)=\int_{\iota^{-1}\left(z_{0}\right) \cap \partial_{m} D} M_{D}(x, a) v_{w}(d a) .
$$

Therefore $\iota^{-1}\left(z_{0}\right) \cap \partial_{m} D \neq \emptyset$. Fix $w_{0} \in \iota^{-1}\left(z_{0}\right) \cap \partial_{m} D$ and let $w_{1}, w_{2} \in \iota^{-1}\left(z_{0}\right)$. Since $M_{D}\left(x, w_{1}\right)$ and $M_{D}\left(x, w_{0}\right)$ are harmonic with respect to $X$ in $D$ (Proposition 5.1) and vanish continuously on $\partial D \backslash\left\{z_{0}\right\}$ (Corollary 5.3), from Lemma 5.5 we know that there exists $c_{3}$ such that

$$
M_{D}\left(x, w_{1}\right) \leq c_{3} \frac{M_{D}\left(x_{0}, w_{1}\right)}{M_{D}\left(x_{0}, w_{0}\right)} M_{D}\left(x, w_{0}\right)=c_{3} M_{D}\left(x, w_{0}\right) .
$$

The minimal harmonicity of $M_{D}\left(\cdot, w_{0}\right)$ implies that $M_{D}\left(x, w_{1}\right)=c_{3} M_{D}\left(x, w_{0}\right)$. But they agree on $x=x_{0}$. So $M_{D}\left(\cdot, w_{1}\right)=M_{D}\left(\cdot, w_{0}\right)$. The same argument shows that $M_{D}\left(\cdot, w_{2}\right)=M_{D}\left(\cdot, w_{0}\right)$, thus $M_{D}\left(\cdot, w_{2}\right)=M_{D}\left(\cdot, w_{1}\right)$, which implies that $w_{1}=w_{2}$. Therefore $\iota$ is one to one. The above argument also says that every $w \in$ $\iota^{-1}(\partial D)$ is a minimal Martin boundary point. Since $\iota$ is onto and $\iota\left(\partial_{M} D\right)=\partial D$ (Lemma 5.2), every $w \in \partial_{M} D$ is a minimal Martin boundary point of $X^{D}$. Therefore $\partial_{m} D=\partial_{M} D$

Lemmas 5.2 and 5.6 imply there exists a homeomorphism between $\partial_{m} D \cup D$ and $\bar{D}$ which is an identity map in $D$. Therefore we arrive the following result.

Theorem 5.7 There is a one-to-one correspondence between the minimal Martin boundary $\partial_{m} D$ and the Euclidean boundary $\partial D$.

\section{Extensions to non-symmetric diffusions with measure-valued drifts}

In this section, we will briefly indicate that the results of this paper remain valid for non-symmetric diffusions with measure-valued drifts. To give a precise definition of a diffusion with drift $\mu$ in $\mathbf{K}_{d, 1}$, we let $\varphi(x)$ and $\varphi_{n}(x)$ be as in Section 1 . For $1 \leq i \leq d$ and $n=1,2, \ldots$, define

$$
U_{n}^{i}(x)=\int \varphi_{n}(x-y) \mu^{i}(d y) .
$$

Put $U_{n}(x)=\left(U_{n}^{1}(x), \ldots, U_{n}^{d}(x)\right)$. Let $L$ be either $L_{1}$ or $L_{2}$ where

$$
L_{1}:=\frac{1}{2} \sum_{i, j=1}^{d} \partial_{i}\left(a_{i j} \partial_{j}\right) \text { and } L_{2}:=\frac{1}{2} \sum_{i, j=1}^{d} a_{i j} \partial_{i} \partial_{j}
$$

with $\mathbf{A}:=\left(a_{i j}\right)$ being $C^{1}$ and uniformly elliptic. 
Definition 6.1 Suppose $\mu=\left(\mu^{1}, \ldots, \mu^{d}\right)$ is such that each $\mu^{i}$ is a signed measure on $\mathbf{R}^{d}$ belonging to the Kato class $\mathbf{K}_{d, 1}$. A diffusion with drift $\mu$ is a family of probability measures $\left\{\mathbf{P}_{x}: x \in \mathbf{R}^{d}\right\}$ on $C\left([0, \infty), \mathbf{R}^{d}\right)$, the space of continuous $\mathbf{R}^{d}$-valued functions on $[0, \infty)$, such that under each $\mathbf{P}_{x}$ we have

$$
Z_{t}=x+Y_{t}+A_{t}
$$

where

(a) $\quad A_{t}=\lim _{n \rightarrow \infty} \int_{0}^{t} U_{n}\left(Z_{s}\right) d s$ uniformly in $t$ over finite intervals, where the convergence is in probability;

(b) there exists a subsequence $\left\{n_{k}\right\}$ such that

$$
\sup _{k} \int_{0}^{t}\left|U_{n_{k}}\left(Z_{s}\right)\right| d s<\infty
$$

almost surely for each $t>0$;

(c) $Y_{t}$ is a diffusion in $\mathbf{R}^{d}$ starting from the origin with generator $L$.

When $A$ is symmetric, the existence and uniqueness of $Z$ were established in [6] (see Remark 6.1 in [6]). In [21], we discussed Brownian motions with the drifts $\mu \in \mathbf{K}_{d, 1}$. when $A$ is symmetric, all of results can be extended to diffusions with the drift $\mu$ due to the estimates on the density and its gradients for the diffusion $Y$ (see [24] for divergence case and [17] for non-divergence case) and the continuities of the density and its gradients. We state the extensions of the main results in [21] without proofs since they will be almost the same.

$Z$ has a transition density $r(t, x, y)$ which is continuous on $(0, \infty) \times \mathbf{R}^{d} \times \mathbf{R}^{d}$ and that there exist positive constants $C_{i}, i=1, \ldots, 9$ such that

$$
C_{1} e^{-C_{2} t} t^{-\frac{d}{2}} e^{-\frac{C_{3}|x-y|^{2}}{2 t}} \leq r(t, x, y) \leq C_{4} e^{C_{5} t} t^{-\frac{d}{2}} e^{-\frac{C_{6}|x-y|^{2}}{2 t}}
$$

and

$$
\left|\nabla_{x} r(t, x, y)\right| \leq C_{7} e^{C_{8} t} t^{-\frac{d+1}{2}} e^{-\frac{C_{9}|x-y|^{2}}{2 t}}
$$

for all $(t, x, y) \in(0, \infty) \times \mathbf{R}^{d} \times \mathbf{R}^{d}$.

In fact, when we deal with $Z^{D}$ (the process obtained by killing $Z$ upon exiting from $D$ ) for a bounded domain $D$, the symmetry assumption is unnecessary by the following simple reduction; Let $b_{i j}:=\frac{1}{2}\left(a_{i j}+a_{j i}\right)$ and note that

$$
\sum_{i, j=1}^{d} a_{i j} \partial_{i} \partial_{j}=\sum_{i, j=1}^{d} b_{i j} \partial_{i} \partial_{j}=\sum_{i, j=1}^{d} \partial_{i}\left(b_{i j} \partial_{j}\right)-\sum_{i, j=1}^{d}\left(\partial_{i} b_{i j}\right) \partial_{j}
$$


and

$$
\begin{aligned}
\sum_{i, j=1}^{d} \partial_{i}\left(a_{i j} \partial_{j}\right) & =\sum_{i, j=1}^{d} a_{i j} \partial_{i} \partial_{j}+\sum_{i, j=1}^{d}\left(\partial_{i} a_{i j}\right) \partial_{j} \\
& =\sum_{i, j=1}^{d} \partial_{i}\left(b_{i j} \partial_{j}\right)+\sum_{i, j=1}^{d} \frac{1}{2} \partial_{i}\left(a_{i j}-a_{j i}\right) \partial_{j}
\end{aligned}
$$

Let $Y_{t}$ be the diffusion in $\mathbf{R}^{d}$ with generator

$$
\frac{1}{2} \sum_{i, j=1}^{d} \partial_{i}\left(b_{i j} \partial_{j}\right)
$$

Since, for any bounded domain $D$,

$$
\begin{aligned}
& \left(\left.\sum_{i=1}^{d} \frac{1}{2} \partial_{i} b_{i 1}\right|_{D}, \ldots,\left.\sum_{i=1}^{d} \frac{1}{2} \partial_{i} b_{i d}\right|_{D}\right) \text { and } \\
& \left(\left.\sum_{i=1}^{d} \frac{1}{4} \partial_{i}\left(a_{i 1}-a_{1 i}\right)\right|_{D}, \ldots,\left.\sum_{i=1}^{d} \frac{1}{4} \partial_{i}\left(a_{i d}-a_{1 d}\right)\right|_{D}\right)
\end{aligned}
$$

are in $\mathbf{K}_{d, 1}$, we construct $Z_{t}$ with a drift which is either

$$
\left(\mu^{1}+\left.\frac{1}{2} \sum_{i=1}^{d} \partial_{i} b_{i 1}\right|_{D} d x, \ldots, \mu^{d}+\left.\frac{1}{2} \sum_{i=1}^{d} \partial_{i} b_{i d}\right|_{D} d x\right)
$$

or

$$
\left(\mu^{1}+\left.\frac{1}{4} \sum_{i=1}^{d} \partial_{i}\left(a_{i 1}-a_{1 i}\right)\right|_{D} d x, \ldots, \mu^{d}+\left.\frac{1}{4} \sum_{i=1}^{d} \partial_{i}\left(a_{i d}-a_{1 d}\right)\right|_{D} d x\right)
$$

as in the Definition 6.1. Then the generator of the killed diffusion process $Z^{D}$ in $D$ can be informally written as $L+\mu \cdot \nabla$ where $L$ is either

$$
\frac{1}{2} \sum_{i, j=1}^{d} \partial_{i}\left(a_{i j} \partial_{j}\right) \text { or } \frac{1}{2} \sum_{i, j=1}^{d} a_{i j} \partial_{i} \partial_{j}
$$

with $\mathbf{A}:=\left(a_{i j}\right)$ being $C^{1}$ and uniformly elliptic but not necessarily symmetric.

Using the estimates on the density and its gradients for the diffusion $Y^{D}$ for divergence case (see [24]) and the continuities of the density and its gradients, the proof of the following estimates is the same as the one in [21]: for every bounded $C^{1,1}$ domain 
$D, Z^{D}$ has a density $r^{D}$ which is continuous on $(0, \infty) \times D \times D$ and that for any $T>0$, there exist positive constants $C_{i}, i=10, \ldots, 14$, such that

$$
C_{10} \psi_{D}(t, x, y) t^{-\frac{d}{2}} e^{-\frac{C_{11}|x-y|^{2}}{t}} \leq r^{D}(t, x, y) \leq C_{12} \psi_{D}(t, x, y) t^{-\frac{d}{2}} e^{-\frac{C_{13}|x-y|^{2}}{t}}
$$

and

$$
\left|\nabla_{x} r^{D}(t, x, y)\right| \leq C_{14}\left(1 \wedge \frac{\rho(y)}{\sqrt{t}}\right) t^{-\frac{d+1}{2}} e^{-\frac{C_{13}|x-y|^{2}}{t}}
$$

for all $(t, x, y) \in(0, T] \times D \times D$.

With these estimates (6.1)-(6.4) in hand, the arguments of this paper can be generalized to the present setting with obvious modifications. We omit all the details.

Remark 6.2 In the forthcoming papers [19,20], we will discuss on the intrinsic ultracontractivity of the semigroup of $Z^{D}$ and the dual processs of $Z^{D}$, respectively.

\section{References}

1. Ancona, A.: Principe de Harnack à la frontir̀e et thórme de Fatou pour un opŕateur elliptique dans un domaine lipschitzien. Ann. Inst. Fourier (Grenoble) 28(4), 169-213 (1978)

2. Aronson, D.G.: Non-negative solutions of linear parabolic equations. Ann. Scuola Norm. Sup. Pisa 22, 607-694 (1988)

3. Bass, R.F.: Probabilistic Techniques in Analysis. Springer, Heidelberg (1995)

4. Bass, R.F., Burdzy, K.: A boundary Harnack principle in twisted Hölder domains. Ann. Math. 134(2), 253-276 (1991)

5. Bass, R.F., Burdzy, K.: A probabilistic proof of the boundary Harnack principle. Seminar on Stochastic Processes (1989), 1-16, Birkhäuser, Boston (1990)

6. Bass, R.F., Chen, Z.-Q.: Brownian motion with singular drift. Ann. Probab. 31(2), $791-817$ (2003)

7. Blumenthal, R.M., Getoor, R.K.: Markov Processes and Potential Theory. Academic, New York (1968)

8. Caffarelli, L., Fabes, E., Mortola, S., Salsa, S.: Boundary behavior of nonnegative solutions of elliptic operators in divergence form. Indiana Univ. Math. J. 30(4), 621-640 (1981)

9. Chen, Z.-Q., Williams, R.J., Zhao, Z.: On the existence of positive solutions for semilinear elliptic equations with singular lower order coefficients and Dirichlet boundary conditions. Math. Ann. 315(4), 735-769 (1999)

10. Chung, K.L.: Lectures from Markov processes to Brownian motion. Springer, New York (1982)

11. Chung, K.L., Zhao, Z.X.: From Brownian motion to Schrödinger's equation. Springer, Berlin (1995)

12. Chung, K.L., Liao, M., Rao, K.M.: Duality under a new setting. Seminar on stochastic processes (1983), pp. 23-38. Birkhaüser, Boston (1984)

13. Chung, K.L., Rao, K.M.: A new setting for potential theory. Ann. Inst. Fourier 30, 167-198 (1980)

14. Dahlberg, B.: Estimates of harmonic measure. Arch. Rational Mech. Anal. 65(3), 275-288 (1977)

15. Davies, E.B.: The equivalence of certain heat kernel and Green function bounds. J. Funct. Anal. 71, 88-103 (1987)

16. Fabes, E., Garofalo, N., Mari'n-Malave, S., Salsa, S.: Fatou theorems for some nonlinear elliptic equations. Rev. Mat. Iberoam. 4(2), 227-251 (1988)

17. Garroni, M.G., Menaldi, J.L.: Green Functions for Second Order Parabolic Integro-differential Problems. Longman, Harlow (1992)

18. Kim, P., Song, R.: Estimates on Green functions and the Schrödinger-type equations for nonsymmetric diffusions with measure-valued drifts. J. Math. Anal. Appl. 332(1), 57-80 (2007)

19. Kim, P., Song, R.: Intrinsic ultracontractivity of non-symmetric diffusions with measure-valued drifts and potentials. Preprint (2006)

20. Kim, P., Song, R.: On dual processes of non-symmetric diffusions with measure-valued drifts. Preprint (2006)

21. Kim, P., Song, R.: Two-sided estimates on the density of Brownian motion with singular drift. Ill. J. Math. 50(3), 635-688 (2006) 
22. Martin, R.S.: Minimal positive harmonic functions. Trans. Am. Math. Soc. 49, 137-172 (1941)

23. Pop-Stojanović, Z.R.: Continuity of excessive harmonic functions for certain diffusions. Proc. Am. Math. Soc. 103(2), 607-611 (1988)

24. Riahi, L.: Comparison of Green functions and harmonic measure of parabolic operators. Potential Anal. 23(4), 381-402 (2005)

25. Taylor, J.C.: The Martin compactification associated with a second order strictly elliptic partial differential operator on a manifold $M$. In: Topics in probability and Lie groups: boundary theory, pp. 153-202, CRM Proc. Lecture Notes, 28, Am. Math. Soc., Providence, RI (2001)

26. Wu, J.M.: Comparisons of kernel functions, boundary Harnack principle and relative Fatou theorem on Lipschitz domains. Ann. Inst. Fourier (Grenoble) 28(4), 147-167 (1978)

27. Zhang, Q.S.: Gaussian bounds for the fundamental solutions of $\nabla(A \nabla u)+B \nabla u-u_{t}=0$. Manuscr. Math. 93, 381-390 (1997)

28. Zhang, Q.S.: The boundary behavior of heat kernels of Dirichlet Laplacians. J. Differ. Equ. 182, 416-430 (2002) 